\title{
HYDRODYNAMIC LIMIT EQUATION FOR A LOZENGE TILING GLAUBER DYNAMICS
}

\author{
BENOÎT LASLIER AND FABIO LUCIO TONINELLI
}

\begin{abstract}
We study a reversible continuous-time Markov dynamics on lozenge tilings of the plane, introduced by Luby et al. 14. Single updates consist in concatenations of $n$ elementary lozenge rotations at adjacent vertices. The dynamics can also be seen as a reversible stochastic interface evolution. When the update rate is chosen proportional to $1 / n$, the dynamics is known to enjoy especially nice features: a certain Hamming distance between configurations contracts with time on average [14] and the relaxation time of the Markov chain is diffusive [20], growing like the square of the diameter of the system. Here, we present another remarkable feature of this dynamics, namely we derive, in the diffusive time scale, a fully explicit hydrodynamic limit equation for the height function (in the form of a non-linear parabolic PDE). While this equation cannot be written as a gradient flow w.r.t. a surface energy functional, it has nice analytic properties, for instance it contracts the $\mathbb{L}^{2}$ distance between solutions. The mobility coefficient $\mu$ in the equation has non-trivial but explicit dependence on the interface slope and, interestingly, is directly related to the system's surface free energy. The derivation of the hydrodynamic limit is not fully rigorous, in that it relies on an unproven assumption of local equilibrium.
\end{abstract}

2010 Mathematics Subject Classification: 60K35, 82C20, 52C20

Keywords: Lozenge tilings, Glauber dynamics, Hydrodynamic limit, Local equilibrium

\section{INTRODUCTION}

The large-scale time evolution of interfaces separating different thermodynamic phases is a classical subject in statistical mechanics. A first natural goal is that of obtaining a hydrodynamic limit [10, 18, 7]: take an initial interface configuration that approximates a macroscopic smooth profile, let it evolve via a microscopic Markovian Glauber-type dynamics that, at the lattice level, follows simple local rules and, rescaling time and space properly, prove that the interface converges to the solution of a deterministic PDE. If the two thermodynamic phases separated by the interface are at coexistence, i.e. if they have the same bulk free energy, we expect the correct time rescaling to be diffusive and the limit equation to be a parabolic PDE, in general a non-linear one, of the form

$$
\partial_{t} \phi(x, t)=-\mu(\nabla \phi(x, t)) \frac{\delta F[\phi]}{\delta \phi(x, t)} .
$$

Here, $F[\phi]$ is the surface tension functional, that is a purely equilibrium quantity, while $\mu(\nabla \phi)>0$ is the (in general slope-dependent) interface mobility coefficient, that depends on the generator of the Markov chain.

To obtain a mathematically simpler model, the interface is often described at the microscopic level by a $d+1$-dimensional height function ("effective interface"), i.e. the 
graph of a function from $\mathbb{Z}^{d}$ to $\mathbb{R}$ (or to $\mathbb{Z}$ in the case of discrete interface models). Here, $d+1$ is the dimension of space where the thermodynamic system of interest lives and of course the physically most relevant case is $d=2$. In the "effective interface" approximation, the internal structure of the two bulk phases is forgotten and the occurrence of interface overhangs is entirely neglected. Despite this somewhat drastic simplification, and despite the fact that the phenomenological picture behind the expected hydrodynamic limit is rather clear [16], most effective interface dynamics remain mathematically intractable and rigorous progress is very limited, especially for $d>1$. One notable exception is that of the Langevin dynamics for the Ginzburg-Landau model with symmetric and strictly convex potential, where a rigorous derivation of the hydrodynamic limit was obtained for any $d \geq 1$ by Funaki and Spohn [6] (see also [15] who extended [6] beyond the case of periodic boundary conditions).

Leaving aside the problem of rigorously proving the hydrodynamic limit, even the more modest goal of guessing the exact form of the limit PDE is in general out of reach, except for lucky exceptions (the Ginzburg-Landau model being one of them) where the dynamics satisfies some form of "gradient condition" [18, 10] which allows to obtain a simple formula for the interface mobility $\mu(\cdot)$, involving only equal-time equilibrium averages.

The goal of the present work is to present a Markov chain for a discrete interface model in dimension $(2+1)$ and to show that it should admit a hydrodynamic limit that is fully explicit and non-trivial $(\mu(\nabla \phi)$ is a non-linear function of the interface slope). We comment below on what is missing in order to turn our arguments into a rigorous proof.

Before introducing the interface dynamics we are interested in, we make a brief detour to motivate the reader. A class of discrete interface dynamics that attracted much attention lately are Glauber dynamics of dimer models, in particular lozenge tilings of the plane [14, 20, 2, 3, 11. Such tilings are in bijection with $(2+1)$-dimensional discrete surfaces obtained as a monotone stacking of elementary cubes in $\mathbb{R}^{3}$, see Figure 1 . Here, "monotone" means that the heights of columns of cubes, indexed by the coordinates $(x, y)$ of their orthogonal projection on the horizontal plane, are weakly decreasing both w.r.t. $x$ and $y$.

The most natural reversible Markov dynamics on such tilings is the one whose elementary moves are rotations by an angle $\pm 60^{\circ}$ (with transition rate 1 ) of three lozenges sharing a common vertex, see Figure 2. This will be called the "single-flip dynamics" in the following. As discussed for instance in [2, 3, the single-flip dynamics coincides with the zero-temperature Glauber dynamics of $+/-$ spin interfaces of the three-dimensional Ising model with zero magnetic field, where spins flip one by one. In terms of monotone stacking of cubes, the dynamics corresponds to adding/removing a cube to/from a column, with transition rate 1 , provided the cube stacking remains monotone after the update. Recently it was proven that, if we restrict the single-flip dynamics to domains of diameter $L$, under certain restrictions on the domain shape the mixing time is of order $O\left(L^{2+o(1)}\right)$ as $L \rightarrow \infty$ [3, 11]. These results support the idea that the correct time-scale to observe a hydrodynamic limit should be diffusive (i.e. that we should rescale time by $L^{2}$ to see a macroscopic evolution) but they are far from being sufficient for proving convergence to a limit PDE. 


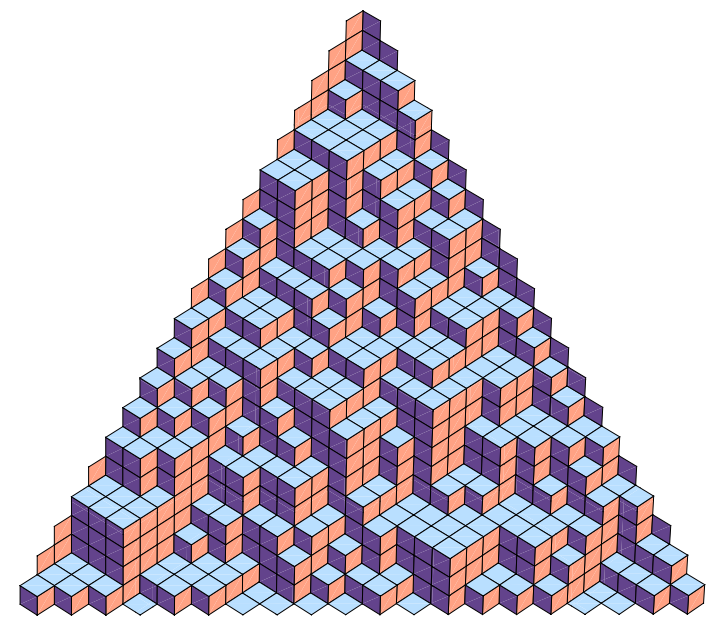

Figure 1. A (portion of) lozenge tiling. Viewing the tiling as a stacking of cubes standing on the horizontal $(x, y)$ plane, note that the height of columns (each with a blue lozenge on top) is weakly decreasing in both $x$ and $y$ directions.

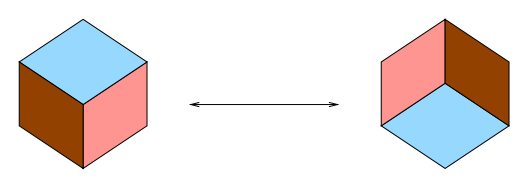

Figure 2. The updates of the single-flip dynamics. Both have transition rate 1 .

In the present work, we study two modifications of the single-flip dynamics, where one allows a number $n \geq 1$ of cubes to be added/removed from a column in each move, again subject to the constraint that the update is legal (i.e. that the resulting configuration is still a monotone stacking of cubes). If the rates are carefully chosen as functions of $n$, the dynamics enjoys much nicer properties than the single-flip one. The first dynamics we will consider is the one where the transition rate of a legal update involving the addition/removal of $n$ cubes is proportional to $1 / n$; in the second dynamics, instead, with rate 1 the height of each column of cubes is resampled from the uniform distribution on all the allowed values it can take given the height of neighboring columns. See Definitions 7 and 8 below for more details. The former dynamics was originally introduced in [14], and the latter in [2]. Both are known to satisfy the special property that the volume difference between two configuration is (on average) decreasing with time, which allows to deduce that the mixing time is at most polynomial in $L$ 14. Moreover, it was proven in [20] that the inverse spectral gap of the dynamics is $O\left(L^{2}\right)$ and that, in special domains, a certain one-dimensional projection of the height function satisfies on average the one-dimensional discrete heat equation.

Here we show that, under a reasonable but unproven assumption of local equilibrium, one can obtain the explicit form of the hydrodynamic limit equation for the height function, see Eqs. 3.3, 3.12 and 3.41 below. Actually, the hydrodynamic equation 
turns out to be the same for both variants of the dynamics. Obtaining such an explicit expression for the hydrodynamic equation is a somewhat surprising fact; indeed, let us stress that in general (for instance, for the single-flip dynamics) the assumption of local equilibrium is not sufficient to guess the limit equation: knowledge of corrections to local equilibrium is also necessary. There is a general heuristic formula [16] for the mobility coefficient $\mu(\nabla \phi)$ which is a variant of Green-Kubo formula. It is given as the sum of two terms, one involving only local averages in the stationary state of slope $\nabla \phi$ and the second involving a time-integral of time-space correlations in the stationary state. The latter term cannot in general be computed as it would require a closed form for spacetime correlations. However in lucky cases (like ours, see Section 4) this term happens to be zero due to a summation by parts at the discrete level.

As we already mentioned, our derivation of the hydrodynamic limit relies on an unproven assumption of local equilibrium. There are various difficulties in proving such assumption, and the direct application of standard entropy techniques (see e.g. [10]) seems out of question, in particular because the stationary measures of the model exhibit long-range correlations. The adaptation of the so-called $H^{-1}$ method employed in [6, 15] looks also challenging: technically a non-trivial difficulty is to get some a-priori control of interface gradients during the evolution (see Remark 17 below for more details). In [6, 15] an important role in this respect was played by strict convexity of the potential, that fails in our case. However, in the case where the system has periodic boundary conditions, in a forthcoming work [12] we manage to overcome these difficulties and to prove rigorously the validity of the hydrodynamic limit.

The hydrodynamic equation has nice analytic features. While it is not in the form of the gradient flow with respect of a surface free energy functional, it can be written in a divergence form (cf. (3.13) ) that allows to show (see Section 3.1.2) that the $\mathbb{L}^{2}$ distance between solutions contracts with time. This is an important point in the program of rigorously proving the convergence towards the hydrodynamic limit equation, and we use this property crucially in our forthcoming work [12] in the periodic boundary condition setting. In fact, the idea of the $H^{-1}$ method is to prove that the $\mathbb{L}^{2}$ distance between the deterministic PDE and the randomly evolving interface stays close to zero at all times. Let us recall briefly how this works in the Ginzburg-Landau model [6, 15. By an entropy production argument [6, Th. 4.1] one shows that at positive times the law of interface gradients is locally close to a certain equilibrium Gibbs measure with an unknown slope. The crucial point is that if the slope is "wrong", i.e. different from that of the solution of the PDE, in which case the random interface has deviated from the deterministic evolution, the derivative of the $\mathbb{L}^{2}$ norm turns out to be negative, which means that the evolution is immediately driven back to the deterministic one (cf. [6, Sec. 5.1]). In turn, the mathematical mechanism behind this fact is the same as the one that guarantees that the $\mathbb{L}^{2}$ between two solutions of the PDE contracts with time.

We will also show that the $\mathbb{L}^{1}$ distance between solutions of the limit PDE is nonincreasing, and decreases only by a boundary term (Section 3.1.1). This is the analogue of the above-mentioned average volume-contraction property of the microscopic dynamics.

Finally let us point out that the exact formula for the hydrodynamic equation leads to some striking identities involving the surface tension (see notably Eq. (3.47) and the 

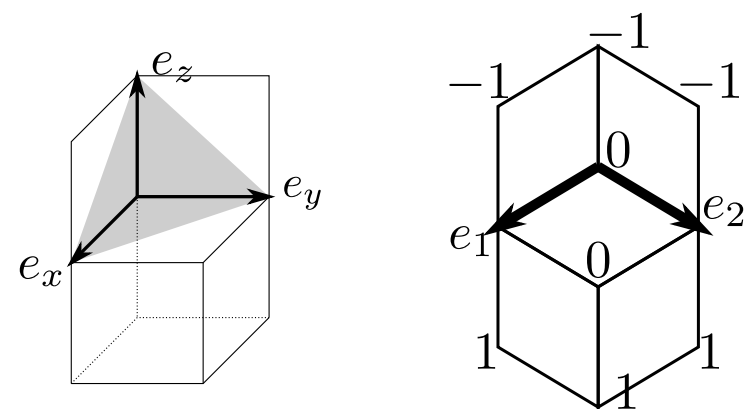

FiguRE 3. Correspondence between monotone surface and lozenge tiling. Left: A monotone surface seen as a subset of $\mathbb{R}^{3}$, with the axes of $\mathbb{R}^{3}$. The grey triangle lies in the plane $P_{111}$. Right: The corresponding lozenge tiling with its height function and the vectors $e_{1}$ and $e_{2}$.

discussion in Remark 16) for which it would be very interesting to find a probabilistic interpretation.

The work is organized as follows. In Section 2 we introduce precisely the model and the dynamics. The hydrodynamic equation is given (in two different but equivalent forms) in Section 3, where we also discuss some of its properties, notably volume contraction. In Section 4 we give a first justification for the limit equation, based on linear response theory. In Section 5 instead we derive the hydrodynamic equation under a local equilibrium assumption. Finally, in Section 6 we explain how to perform some useful equilibrium computations.

\section{The MOdEL AND THE DYNAMiCS}

2.1. Monotone surfaces and height function. We start by defining discrete monotone surfaces.

Definition 1. Let $Q$ be the collection of closed squares in $\mathbb{R}^{3}$ of side 1 , with the four vertices in $\mathbb{Z}^{3}$. A discrete (or stepped) monotone surface $\Sigma$ is a connected union of elements of $Q$ that projects bijectively on the $P_{111}$ plane (the linear subspace of $\mathbb{R}^{3}$ of normal vector $(1,1,1))$.

The $P_{111}$ orthogonal projection (denoted $\Pi_{111}$ ) of a square face of $\Sigma$ is a lozenge with angles $\pi / 3$ and $2 \pi / 3$, side-length $\sqrt{2 / 3}$ and three possible orientations: north-west, north-east and horizontal, according to whether the normal vector to the square face in question is $(1,0,0),(0,1,0)$ or $(0,0,1)$. The projection of $\Sigma$ gives therefore a lozenge tiling of $P_{111}$. Vertices of the lozenges are the vertices of a triangular lattice $\mathcal{T}$ of side $\sqrt{2 / 3}$. We will refer to north-west oriented, north-east oriented and horizontal lozenges as lozenges of types 1,2,3 respectively. See Fig. 3 .

Let $e_{x}, e_{y}, e_{z}$ be the usual orthonormal vectors of $\mathbb{R}^{3}$. On the plane $P_{111}$ we introduce unit vectors $e_{1}, e_{2}$ and correspondingly coordinates $u=\left(u_{1}, u_{2}\right)$ as follows: a given reference vertex $u_{0} \in \mathcal{T}$ (for example, the one on which the origin of $\mathbb{Z}^{3}$ projects) has coordinates $(0,0)$ and the vector $e_{1}$ (resp. $\left.e_{2}\right)$, of coordinates $\left(u_{1}, u_{2}\right)=(1,0)$ (resp. $\left.\left(u_{1}, u_{2}\right)=(0,1)\right)$ is the vector from $u_{0}$ to its nearest neighbor in direction $e^{-5 i \pi / 6}$ (resp. 
$\left.e^{-i \pi / 6}\right)$. That is, $e_{1}=\Pi_{111}\left(e_{x}\right), e_{2}=\Pi_{111}\left(e_{y}\right)$. Note that with this choice of coordinates, triangular faces of $\mathcal{T}$ have side-length 1 and not $\sqrt{2 / 3}$.

In order to turn the correspondence between stepped surfaces and lozenge tilings into a bijection, we impose that $(0,0,0) \in \Sigma$.

Given a discrete monotone surface $\Sigma$, the height function $h=h_{\Sigma}: \mathcal{T} \mapsto \mathbb{Z}$ is defined as follows: $h\left(u_{1}, u_{2}\right)$ equals minus the height with respect to the horizontal plane (i.e. minus the $z$ coordinate) of the point $p \in \Sigma$ that projects on $\left(u_{1}, u_{2}\right)$, i.e. such that $\Pi_{111}(p)=u_{1} e_{1}+u_{2} e_{2}$. Of course $h(0,0)=0$ since we imposed $(0,0,0) \in \Sigma$. The reason for the minus sign is that otherwise the interface gradients would be given by minus the lozenge densities (see Remark 2 just below), which would lead to less readable formulas later. The height function can be naturally extended to the whole plane by linear interpolation in each face of $\mathcal{T}$.

Remark 2. Observe that when one moves by one lattice step in $\mathcal{T}$ along the $+e_{1}$ or $+e_{2}$ directions the height function increases by 1 if one crosses a lozenge, and is unchanged if one moves along the edge of a lozenge. When instead one moves by a lattice step upward in the vertical direction (i.e. by $-e_{1}-e_{2}$ ), the height function is unchanged if one crosses a lozenge, and decreases by 1 if one moves along the edge of a lozenge.

We will be interested in dynamics in finite domains. For $L=1,2, \ldots$ let $U_{L}$ be a simply connected, bounded union of triangular faces of $\mathcal{T}$ that can be tiled by lozenges, and let $\partial U_{L}$ be its boundary, seen as a collection of edges of $\mathcal{T}$. Assume that the site $(0,0)$ where height is fixed to zero is on $\partial U_{L}$.

Call $\Omega_{U_{L}}$ the set of lozenge tilings of $U_{L}$ and $\eta$ the generic element of $\Omega_{U_{L}}$. Remark that the height function on $\partial U_{L}$ is independent of the configuration $\eta \in \Omega_{U_{L}}$. If wished, one can imagine that $\eta$ is extended to a lozenge tiling $\eta^{\prime}$ of the whole plane, just by completing $\eta$ with a tiling $\eta_{0}$ of $P_{111} \backslash U_{L}$, fixed once and for all. From this point of view, $\Omega_{U_{L}}$ can be identified with the set of monotone surfaces $\Sigma$ such that $(0,0,0) \in \Sigma$ and such that the projection of $\Sigma$ restricted to $P_{111} \backslash U_{L}$ coincides with $\eta_{0}$.

We will assume from now on that $U_{L}$ has a scaling limit in the following sense:

Assumption 3. As $L \rightarrow \infty,(1 / L) U_{L}$ tends in Hausdorff distance to a bounded simply connected closed domain $U \subset \mathbb{R}^{2}$ with smooth boundary, and the graph of the function

$$
u \in \frac{1}{L} \partial U_{L} \mapsto \frac{1}{L} h_{\eta^{\prime}}(u L) \in \mathbb{R}
$$

tends to the graph of a continuous function $\phi: \partial U \mapsto \mathbb{R}$. Moreover, there exists a $C^{1}$ function $\psi: U \mapsto \mathbb{R}$ such that $\psi=\phi$ on $\partial U$ and that

$$
\nabla \psi(u)=\left(\partial_{u_{1}} \psi, \partial_{u_{2}} \psi\right)(u) \in \mathbb{T} \quad \forall u \in U
$$

where $\mathbb{T} \subset \mathbb{R}^{2}$ is the open triangle with vertices $(0,0),(1,0),(0,1)$.

To understand the condition (2.1), recall that

Theorem 4. [5] Given $\psi: U \mapsto \mathbb{R}$ satisfying (2.1) and $\left.\psi\right|_{\partial U}=\phi$,

$$
\lim _{\delta \rightarrow 0} \lim _{L \rightarrow \infty} \frac{1}{L^{2}} \ln \left|\left\{\eta \in \Omega_{U_{L}}: \sup _{u \in U}\left|L^{-1} h_{\eta}(u L)-\psi(u)\right| \leq \delta\right\}\right|=-\int_{U} \sigma(\nabla \psi) d u
$$


where $\ln$ denotes the natural logarithm and, for $\rho=\left(\rho_{1}, \rho_{2}\right)$,

$$
\sigma(\rho)=\left\{\begin{array}{cr}
\frac{1}{\pi}\left[\Lambda\left(\pi \rho_{1}\right)+\Lambda\left(\pi \rho_{2}\right)+\Lambda\left(\pi\left(1-\rho_{1}-\rho_{2}\right)\right)\right] \leq 0, & \rho \in \mathbb{T} \cup \partial \mathbb{T} \\
+\infty & \text { otherwise }
\end{array}\right.
$$

with

$$
\Lambda(\theta)=\int_{0}^{\theta} \ln (2 \sin (t)) d t
$$

Observe that the argument of $\Lambda$ in $(2.3)$ is positive, since $\rho_{1}, \rho_{2}, \rho_{3}:=1-\rho_{1}-\rho_{2} \geq 0$ if $\rho \in \mathbb{T}$. The function $\sigma$ is real analytic and strictly negative in $\mathbb{T}$, vanishes when $\rho \in \partial \mathbb{T}$ and its gradient diverges when $\partial \mathbb{T}$ is approached. The condition (2.1) guarantees in particular that the cardinality of $\Omega_{U_{L}}$ is exponentially large in $L^{2}$, i.e. the entropy per unit area is positive.

In view of the definition of height function, one should think of $\rho_{i}$ as the density of lozenges of types $i=1,2,3$. We however emphasize that making point-wise sense of this intuition is a delicate problem.

2.2. Translation-invariant Gibbs states. It is well known 8 that for every $\rho \in \mathbb{T}$ there exists an unique translation invariant, ergodic Gibbs state on the set of lozenge tilings of the plane, such that the density of lozenges of type $i$ is $\rho_{i}$. Such measures have the following explicit form. Let the hexagonal lattice $\mathcal{H}$ be the dual of $\mathcal{T}$ and color its vertices black/white in an alternate way, see Figure 4. A white vertex is given the same coordinate $x=\left(x_{1}, x_{2}\right)$ as the black vertex just to its right, and the coordinates $x_{1}, x_{2}$ correspond to the axes $e_{1}, e_{2}$ introduced above. There exists a natural bijection between lozenge tilings $\eta$ of the plane and perfect matchings $M$ of $\mathcal{H}$, see Figure 4 .

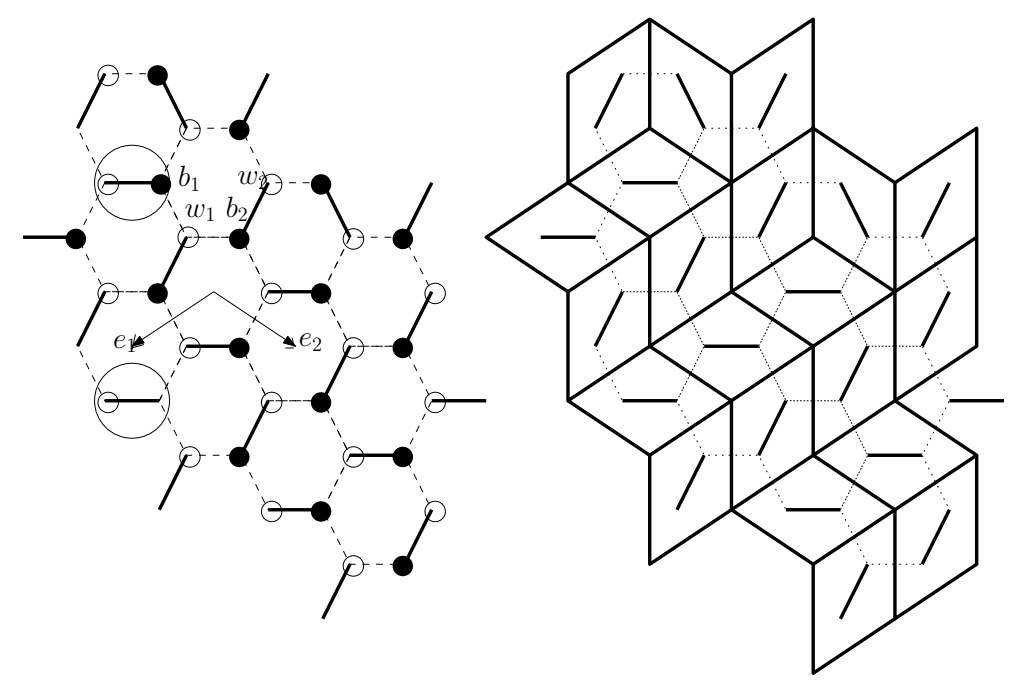

FiguRE 4. The bijection between perfect matching of $\mathcal{H}$ (left) and lozenge tiling (right). The vertices $w_{1}, b_{1}, w_{2}, b_{2}$ have coordinates respectively $(0,0),(0,-1),(-1,0),(0,0)$. The two encircled horizontal dimers ("particles") have the same column coordinate $u_{1}-u_{2}$ and vertical coordinate differing by 2 . 
Take a triangle with angles $\theta_{i}=\pi \rho_{i}, i=1,2,3$ and let $k_{i}$ be the length of the side opposite to $\theta_{i}$. Given an edge $e$ of $\mathcal{H}$, say that it is of type 1,2 or 3 if it is north-west, north-east oriented or horizontal and let $K(e):=k_{i}$. Then, given an integer $n$ and edges $e_{i}, i \leq n$ such that the white (resp. black) vertex of $e_{i}$ is $w_{i}$ (resp. $b_{i}$ ), one has [8]

$$
\pi_{\rho}\left(e_{1}, \ldots, e_{n} \in M\right)=K\left(e_{1}\right) \ldots K\left(e_{n}\right) \operatorname{det}\left(\left\{K^{-1}\left(w_{i}, b_{j}\right)\right\}_{i, j=1, \ldots, n}\right)
$$

where, if $w$ has coordinates $\left(x_{1}, x_{2}\right)$ and $b$ has coordinates $\left(y_{1}, y_{2}\right)$,

$$
K^{-1}(w, b)=\frac{1}{(2 \pi i)^{2}} \oint \frac{d z}{z} \oint \frac{d w}{w} \frac{z^{x_{2}-y_{2}} w^{y_{1}-x_{1}}}{k_{3}+k_{1} z+k_{2} w}
$$

and the integrals runs over the torus $\left\{(z, w) \in \mathbb{C}^{2}:|z|=|w|=1\right\}$. Note that (2.4) is unchanged if all $k_{i}$ are multiplied by a common factor. In particular, if $e=(w, b)$ is an edge of type $i$ one has

$$
\rho_{i}=k_{i} K^{-1}(w, b) .
$$

2.3. Particles. We have seen that the $P_{111}$ projection of any discrete monotone surface gives a lozenge tiling $\eta$ of the plane. Horizontal lozenges will be called "particles" and will be given a label $b$. To each particle will be associated a "vertical position" $n(b)$, defined as

$$
n(b)=\frac{u_{1}+u_{2}}{2},
$$

with $\left(u_{1}, u_{2}\right)$ the coordinates of the upper corner of the particle (horizontal lozenge), as well as a "horizontal position" (or "column coordinate")

$$
c(b)=u_{1}-u_{2},
$$

see Figure 4 . Note that $n(b) \in \mathbb{Z}$ if $c(b) \in 2 \mathbb{N}$ (i.e. if the column containing $b$ has the same parity as the column containing the vertex $(0,0))$, and $n(b) \in \mathbb{Z}+1 / 2$ otherwise.

Recalling Remark 2, observe that when the vertical coordinate of a particle $b$ changes by $\pm|n|$, there are $n$ vertices in the triangular lattice $\mathcal{T}$ where the height changes by \pm 1 .

It is well known (and easy to check) that a lozenge tiling of the plane is uniquely determined by the particle positions, provided that there is at least one particle per column, which we will assume henceforth. Recall that the height function on $\partial U_{L}$ is independent of the configuration $\eta \in \Omega_{U_{L}}$. From the definition of height function, we deduce that for each column $i$, the number of particles on column $i$ that are in $U_{L}$ is the same for every $\eta \in \Omega_{U_{L}}$. Actually the whole tiling $\eta$ is uniquely determined (once $U_{L}$ and $\eta_{0}$ on $P_{111} \backslash U_{L}$ are given) by the positions of the particles in $U_{L}$.

It is also well known and easy to check that particle positions satisfy the following interlacement properties: if $b, b^{\prime}$ are two particles on the same column $i=c(b)=c\left(b^{\prime}\right)$ with $n(b)<n\left(b^{\prime}\right)$ and if there is no particle $b^{\prime \prime}$ on column of index $i$ with $n(b)<n\left(b^{\prime \prime}\right)<$ $n\left(b^{\prime}\right)$ then there is a unique particle $b^{\text {right }}$ (resp. $b^{\text {left }}$ ) in column $i+1$ (resp. $i-1$ ) such that $n(b)<n\left(b^{\text {right }}\right)<n\left(b^{\prime}\right)\left(\right.$ resp. $\left.n(b)<n\left(b^{\text {left }}\right)<n\left(b^{\prime}\right)\right)$.

In the study of our interface dynamics we will need the following two definitions:

Definition 5. Given $\eta \in \Omega_{U_{L}}$ and a particle $b$ in $U_{L}$, we let $n^{+}(b), n^{-}(b)$ be the largest/smallest possible vertical position that particle $b$ can take in any configuration 
$\eta^{\prime} \in \Omega_{L}$ such that all particles other than $b$ have the same position as in $\eta$. See Figure 5 (a). Of course $n^{ \pm}(b)$ is a function of $\eta$. Also, we call $I(b)=\left\{n^{-}(b), \ldots, n^{+}(b)\right\}$.

(a)

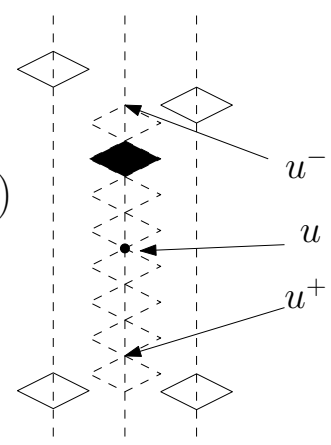

(b)

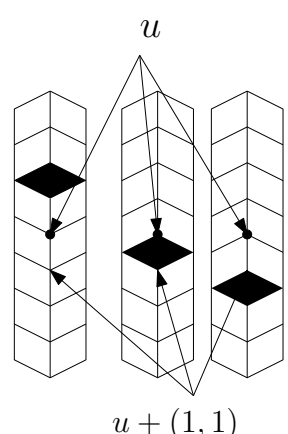

(c)

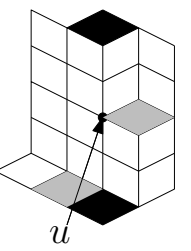

FiguRE 5. (a): For the black particle $b$ one has $n^{+}(b)=\left(u_{1}^{+}+u_{2}^{+}\right) / 2$ and $n^{-}(b)=\left(u_{1}^{-}+u_{2}^{-}\right) / 2$ (recall that vertical positions are measured w.r.t. $-e_{1}-e_{2}$, which is why $u^{+}$appears below $u^{-}$in the drawing). Further moves are prevented by the four white particles. The event $X(u)$ is verified here and particle $b^{(u)}$ is the black one. (b): According to the position of $b^{(u)}$ w.r.t. $u$, the edge $(u, u+(1,1))$ can either cross the particle $b^{(u)}$ (mid drawing) or be the common edge of two lozenges, of types 1 and 2 (left and right drawing). (c) In this configuration, the event $X(u)$ is not realized. None of the particles (drawn in black) in the column of $u$ can take vertical position $\left(u_{1}+u_{2}\right) / 2$ without pushing particles (in gray) in neighboring columns.

Definition 6. Given a lozenge tiling of the plane and $u=\left(u_{1}, u_{2}\right) \in \mathcal{T}$ we call $X(u)$ the event that there exists a particle $b^{(u)}$ with column coordinate $u_{1}-u_{2}$ such that

$$
n^{-}\left(b^{(u)}\right) \leq \frac{u_{1}+u_{2}}{2} \leq n^{+}\left(b^{(u)}\right),
$$

see Figure 5 (a). (Such particle is necessarily unique). The particle $b^{(u)}$ can be moved to position $\left(u_{1}+u_{2}\right) / 2$ without moving any other particle.

The event $X(u)$ is equivalently the event that the edge $u, u+(1,1)$ of $\mathcal{T}$ is either the common side of two lozenges, one of type 1 and one of type 2 , or it crosses a lozenge of type 3, see Figure 5 (b). In the latter case, one has $n\left(b^{(u)}\right)=\left(u_{1}+u_{2}\right) / 2$. Therefore, the event $X(u)$ is not realized when the edge $u, u+(1,1)$ is the common side of two lozenges, both of type 1 or both of type 2 (e.g. Fig. 5 (c)).

2.3.1. Interpretation of $\rho$ in terms of particles. One can give an interpretation, purely in terms of interlaced particles, to the two parameters $\rho_{1}, \rho_{2}$ labelling the Gibbs measures $\pi_{\rho}$. First of all, $1-\rho_{1}-\rho_{2}$ is the density of particles in any given column. The difference $\rho_{1}-\rho_{2}$ corresponds to an asymmetry parameter as follows. Look at a column, say the one labelled 0 , and call $\left\{n_{i}\right\}_{i \in \mathbb{Z}}$ the vertical positions of its particles $\left\{b_{i}\right\}_{i \in \mathbb{Z}}$, ordered so that $n_{i}<n_{i+1}$. Given particles $b_{i}$ and $b_{i+1}$, let $b_{i}^{\prime}$ be the unique particle in column 1 
whose vertical position $n_{i}^{\prime}$ satisfies $n_{i}<n_{i}^{\prime}<n_{i+1}$. Then one has

$$
\frac{\rho_{1}-\rho_{2}}{2}=\lim _{K \rightarrow \infty} \frac{\sum_{i=1}^{K} n_{i}^{\prime}-\frac{n_{i+1}+n_{i}}{2}}{\sum_{i=1}^{K}\left(n_{i+1}-n_{i}\right)}
$$

where the limit holds $\pi_{\rho^{-}}$-almost surely, due to ergodicity of the Gibbs measure. In other words, $\rho_{1}-\rho_{2}$ is a measure of how much $n_{i}^{\prime}$ is biased away from the mid-point $\left(n_{i}+n_{i+1}\right) / 2$.

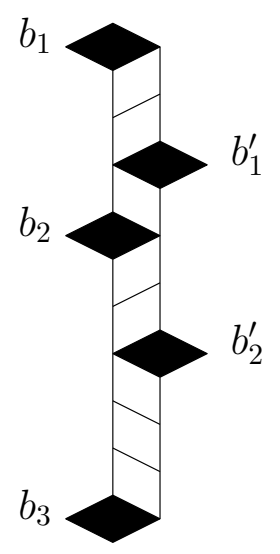

FiguRE 6. If for instance particle $b_{1}$ has position $n_{1}=0$, then $n_{2}=4$, $n_{3}=10$ and $n_{1}^{\prime}=5 / 2, n_{2}^{\prime}=13 / 2$. The number of type- 1 lozenges is $N_{1}^{(2)}=4=\left(n_{2}-n_{1}^{\prime}-1 / 2\right)+\left(n_{3}-n_{2}^{\prime}-1 / 2\right)$.

To see why (2.8) holds, look at Figure 6 r running along column 0 from position $n_{1}$ to $n_{K+1}$, the number of lozenges of type 1 that are adjacent to column 0 to its right is

$$
N_{1}^{(K)}=\sum_{i=1}^{K}\left(n_{i+1}-n_{i}^{\prime}-1 / 2\right)
$$

and the number of lozenges of type 2 is

$$
N_{2}^{(K)}=\sum_{i=1}^{K}\left(n_{i}^{\prime}-n_{i}-1 / 2\right) .
$$

The factors $1 / 2$ keep into account the fact that particle positions in column 0 are integers and those in column 1 are half-integers. One has then

$$
\frac{\rho_{1}-\rho_{2}}{\rho_{1}+\rho_{2}}=\lim _{K} \frac{N_{1}^{(K)}-N_{2}^{(K)}}{N_{1}^{(K)}+N_{2}^{(K)}}=\lim _{K} \frac{\sum_{i=1}^{K}\left(n_{i+1}+n_{i}-2 n_{i}^{\prime}\right)}{\sum_{i=1}^{K}\left(n_{i+1}-n_{i}-1\right)} .
$$

On the other hand,

$$
\frac{\sum_{i=1}^{K}\left(n_{i+1}-n_{i}-1\right)}{\sum_{i=1}^{K}\left(n_{i+1}-n_{i}\right)}=1-\frac{K}{n_{K+1}-n_{1}}
$$

that converges to $1-\rho_{3}=\rho_{1}+\rho_{2}$ since $K$ is the number of particles in column 0 in a segment of length $n_{K+1}-n_{1}$. Equation 2.8 then follows. 
2.4. Dynamics. We will study two continuous-time Markov dynamics on $\Omega_{U_{L}}$. Both are reversible with respect to the uniform measure $\mathbb{P}_{U_{L}}$. We will only define the dynamics in terms of movements of particles but recall that these determine the whole tiling. In the dynamics, only particles in $U_{L}$ can evolve.

Definition 7 (Dynamics I). For any particle $b$ in $U_{L}$ and any $k \in I(b), k \neq n(b), b$ moves to vertical position $k$ with rate $1 /(2|n(b)-k|)$.

This is equivalent to a dynamics introduced by Luby, Randall and Sinclair [14. Let us recall that this dynamics can be used as an auxiliary process to show that the "singleflip" dynamics, where particles are instead allowed to move only to $n(b) \pm 1$ with equal rates (provided $n(b) \pm 1 \in I(b)$ ), has a mixing time and inverse spectral gap that is at most polynomial in $L$.

Definition 8 (Dynamics II). For any particle $b$ and any position $k \in I(b), k \neq n(b), b$ moves to position $n$ with rate $1 /|I(b)|$.

In other words, with rate 1 each particle is redistributed uniformly among its instantaneously available positions. This dynamics was introduced in [2], again as an auxiliary process to analyze the single-flip dynamics.

It is immediate to see that both are reversible w.r.t the uniform measure. We will call $\mathcal{L}^{I}, \mathcal{L}^{I I}$ the generators of the two dynamics. The configuration at time $t$ will be denoted $\eta(t)$ and dependence on the boundary condition as well as the index $I, I I$, that distinguishes between the two dynamics will not be indicated explicitly.

Remark 9. In [19] was defined an irreversible (driven) dynamics on lozenge tilings of the infinite triangular lattice $\mathcal{T}$. In its totally asymmetric version, each particle $b$ jumps to any $k \in I(b), k>n(b)$ with rate 1 . It was proven in [19] that the Gibbs measures $\pi_{\rho}$ is stationary for such driven dynamics (a consistent part of the work consisting in proving that the dynamics is well defined for almost every initial condition sampled from $\pi_{\rho}$ ). We will see (cf. discussion just after Theorem 11) that the two reversible dynamics of Definitions 7 and 8 are not unrelated to the irreversible dynamics of [19].

\section{THE LIMIT HYDRODYNAMIC EQUATION}

Call $\eta=\eta(0)$ the initial condition of the dynamics (actually $\eta$ is a sequence $\left\{\eta_{L}\right\}_{L \in \mathbb{N}}$, but we drop the subscript $L$ ) and $\eta(t)$ the configuration at time $t$ (recall that we do not distinguish between dynamics I and II in the notation). Assume that $\eta$ approximates a smooth profile, i.e. there exists $\psi_{0}$ satisfying (2.1) such that

$$
\lim _{L \rightarrow \infty} \frac{1}{L} h_{\eta}(u L)=\psi_{0}(u)
$$

for every $u \in U$. Let for $t \geq 0, u \in U$

$$
H(u, t)=\frac{1}{L} h_{\eta\left(t L^{2}\right)}(u L) .
$$

On general grounds [16], one expects $H$ to concentrate around a deterministic solution in the sense that there exists some deterministic function $\{\psi(u, t)\}_{u \in U, t \geq 0}$ such that, for every $\epsilon>0$ and $t \geq 0$,

$$
\mathbb{P}(\exists u \in U:|H(u, t)-\psi(u, t)| \geq \epsilon) \stackrel{L \rightarrow \infty}{\rightarrow} 0 .
$$


Furthermore, $\psi$ should follow a non-linear PDE of the form

$$
\left\{\begin{array}{l}
\partial_{t} \psi=\mu(\nabla \psi) \sum_{i, j=1,2} \sigma_{i, j}(\nabla \psi) \frac{\partial^{2}}{\partial_{u_{i}} \partial_{u_{j}}} \psi \\
\psi(u, t)=\psi_{0}(u)
\end{array} \quad \text { if } t=0 \text { or if } u \in \partial U\right.
$$

where $\sigma_{i, j}(\rho):=\partial^{2} \sigma / \partial \rho_{i} \partial \rho_{j}$, with $\sigma$ defined in $(2.3)$, and $\mu(\cdot)$ is a positive function. This equation is of parabolic type, since the Hessian matrix $\left(\sigma_{i, j}(\rho)\right)_{i, j=1,2}$ is strictly positive definite for $\rho \in \mathbb{T}$ (positive definiteness follows from convexity of the surface tension $\sigma$ and strict positivity follows from the fact that the determinant of $\left(\sigma_{i, j}(\rho)\right)_{i, j=1,2}$ equals identically $\pi^{2}$ [9, Th. 5.5], as can also be checked from (3.7) below).

The positive coefficient $\mu(\cdot)$ is called the "mobility" and in general will depend on the microscopic definition of the dynamics. In particular, a priori there is no reason for it to be the same for dynamics I and II, but we will see below that the mobility does in fact coincide in the two cases.

Remark 10. While we see no a priori physical reason why the two mobilities should coincide, let us comment on what is behind this equality. Both dynamics satisfy a form of "gradient condition", that is responsible for the vanishing, thanks to an exact summation by parts, of the term in the Green-Kubo expression (4.17) that involves equilibrium correlations at different times. Then, $\mu(\rho)$ turns out to be proportional to the derivative at time zero of the mean square displacement of a particle, for the process started from the stationary state $\pi_{\rho}$ :

$$
\mu(\rho) \propto \pi_{\rho}\left(\sum_{y: n(b)+y \in I(b)} c_{b, y}(\eta) y^{2}\right),
$$

with $c_{b, y}(\eta)$ the rate at which particle $b$ jumps from position $n(b)$ to $n(b)+y$ in configuration $\eta$. See the first term in (4.17). A simple and explicit computation, using the fact that at equilibrium the position of a particle is uniform given the position of the other particles, shows that, despite the fact that the transition rates are different for dynamics $I$ and II, the average in (3.4) is the same in both cases (see Eq. (4.18)). It would be interesting to understand whether there are other natural transition rates that lead to the same mobility.

Another hint that the two dynamics have common features is that both were designed (in [14] and [2] respectively) to have the property of contracting the mutual volume between two configurations.

The meaning of $(3.3)$ is that, in the diffusive scaling, the interface velocity will be given by the gradient flow associated to the surface tension functional

$$
F(\psi)=\int_{U} \sigma(\nabla \psi) d u
$$

times a certain mobility coefficient $\mu$ that depends on the local slope. Note indeed that

$$
\sum_{i, j=1,2} \sigma_{i, j}(\nabla \psi) \frac{\partial^{2}}{\partial_{u_{i}} \partial_{u_{j}}} \psi=-\frac{\delta F(\psi)}{\delta \psi(u)} .
$$


More explicitly, one finds from 2.3

$$
\begin{aligned}
\sigma_{i, i}(\rho) & =\pi \cot \left(\pi \rho_{i}\right)+\pi \cot \left(\pi\left(1-\rho_{1}-\rho_{2}\right)\right) \\
\sigma_{1,2}(\rho) & =\pi \cot \left(\pi\left(1-\rho_{1}-\rho_{2}\right)\right) .
\end{aligned}
$$

An expression for $\mu(\rho)$ can be obtained from linear response theory. Usually (cf. [16, Section 4] and (4.17) below), such expression is given by the sum of two terms: the first involves the average w.r.t. $\pi_{\rho}$ of a local observable and the second involves the integral over time $t$, ranging from 0 to $\infty$, of the correlations (in the stationary process started from $\pi_{\rho}$ ) between an observable at time 0 and an observable at time $t$. In general, it is not possible to compute the second term explicitly. In lucky cases (e.g. the zerotemperature dynamics of interfaces of the 2D Ising model or the Langevin dynamics of the Ginzburg-Landau effective interface model [16]) the second term vanishes due to a summation by parts. This turns out to be the case also for our dynamics.

The expression for the mobility, provided by linear response theory, is in our case (see Section 4 for a derivation along the lines of [16])

$$
\mu(\rho)=\frac{1}{12} \pi_{\rho}\left[\left(\left|I\left(b^{(0,0)}\right)\right|+1\right) 1_{X(0,0)} 1_{n\left(b^{(0,0)}\right) \neq 0}\right],
$$

for both dynamics I and II. Here, $b^{(0,0)}$ and $X(0,0)$ are just $b^{(u)}, X(u)$ as in Definition 6. and we have arbitrarily chosen $u=(0,0)$ by translation invariance.

In Section 6 we show:

Theorem 11. The r.h.s. of $(3.8)$ equals

$$
\frac{V(\rho)}{2}:=\frac{1}{2} \pi_{\rho}\left[\left(n^{+}\left(b^{(0,0)}\right)+1\right) 1_{X(0,0)} 1_{n\left(b^{(0,0)}\right)<0}\right] .
$$

The advantage of the rewriting $(3.9)$ is that $V(\rho)$ turns out to be nothing but the average interface velocity for the totally asymmetric process defined in Remark 9 , in the stationary measure $\pi_{\rho}$. Namely, let the "total current" $J(0, t)$ denote the number of particles that cross a fixed vertex of the triangular lattice $\mathcal{T}$ in the time interval $[0, t]$ for the asymmetric process. Then [19]

$$
\langle J(0, t)\rangle_{\rho}=t V(\rho),
$$

where $\langle\cdot\rangle_{\rho}$ denotes average w.r.t. the stationary process started from $\pi_{\rho}$. In [4, Th. 2.7] it was proven (with somewhat different notations) that

$$
V(\rho)=\frac{1}{\pi} \frac{\sin \left(\pi \rho_{1}\right) \sin \left(\pi \rho_{2}\right)}{\sin \left(\pi\left(1-\rho_{1}-\rho_{2}\right)\right)}
$$

Recall that $0<\rho_{1}, \rho_{2},\left(1-\rho_{1}-\rho_{2}\right)<1$ and that these three numbers give the average fraction of lozenges of types $1,2,3$ respectively under the measure $\pi_{\rho}$. In conclusion, both for dynamics I and II, the linear response theory mobility defined as in (3.8) equals

$$
\mu(\nabla \psi)=\frac{1}{2 \pi} \frac{\sin \left(\pi \partial_{u_{1}} \psi\right) \sin \left(\pi \partial_{u_{2}} \psi\right)}{\sin \left(\pi\left(1-\partial_{u_{1}} \psi-\partial_{v_{2}} \psi\right)\right)} .
$$

The conjectural explicit form of the hydrodynamic limit equation is then given by (3.3), together with (3.7) and (3.11). 
3.1. Hydrodynamic equation and volume contraction. It goes beyond the scopes of the present work to investigate the existence and regularity of the solutions of (3.3). This might be a non-trivial issue due to the singularity of $\mu(\cdot)$ and $\sigma_{i, j}(\cdot)$ when their argument approaches $\partial \mathbb{T}$. In the following of this section, we will implicitly assume that the domain $U$ and the initial condition $\psi_{0}(\cdot)$ are regular enough that $(3.3)$ admits a unique classical solution $\psi(u, t)$ that is $C^{1}$ in $U \times[0, \infty)$ where we recall that the domain $U$ is closed. In the forthcoming [12] we explain how to extract such existence, uniqueness and smoothness statements from the existing literature (e.g. [13, Chap. XII]).

It is interesting to remark that $(3.3)$ can be rewritten as follows:

$$
\partial_{t} \psi=\operatorname{div}(W \circ \nabla \psi):=\partial_{u_{1}} W^{(1)}\left(\partial_{u_{1}} \psi, \partial_{u_{2}} \psi\right)+\partial_{u_{2}} W^{(2)}\left(\partial_{u_{1}} \psi, \partial_{u_{2}} \psi\right)
$$

where $W=\left(W^{(1)}, W^{(2)}\right)$ and

$$
\begin{aligned}
& W^{(1)}\left(\rho_{1}, \rho_{2}\right)=-\frac{1}{2 \pi} \cot \left(\pi\left(\rho_{1}+\rho_{2}\right)\right) \sin ^{2}\left(\pi \rho_{2}\right)-\frac{\rho_{2}}{4}+\frac{1}{4 \pi} \sin \left(2 \pi \rho_{2}\right) \\
& W^{(2)}\left(\rho_{1}, \rho_{2}\right)=W^{(1)}\left(\rho_{2}, \rho_{1}\right) .
\end{aligned}
$$

(This can be checked via a direct computation, using the definition of $W$, of $\mu$ and the expressions (3.7) for $\sigma_{i, j}$ ). One can also check that the curl of the vector field $W$ is non-zero, which prevents from writing $W$ as the gradient of some function $\hat{\sigma}$ and the equation (3.13) for $\psi$ as the gradient flow w.r.t. the associated functional $-\int_{U} \hat{\sigma}(\nabla \psi) d u$.

The rewriting (3.13) has two interesting consequences, namely contractions in time of both the $\mathbb{L}^{1}$ and $\mathbb{L}^{2}$ distances between solutions. The two phenomena are somewhat different: as we see in a moment, $\mathbb{L}^{1}$ contraction is only a boundary effect, while $\mathbb{L}^{2}$ contraction is a bulk effect.

3.1.1. $\mathbb{L}^{1}$ contraction. By Gauss' theorem, (3.13) implies that the time derivative of the total volume below the surface, $\mathcal{V}_{t}(\psi(\cdot, t)):=\int_{U} \psi(u, t) d u$, is only a boundary term:

$$
\frac{d}{d t} \mathcal{V}(\psi(\cdot, t))=\int_{\partial U} W(\nabla \psi(u, t)) \cdot n d \gamma
$$

with $n$ the exterior normal vector to $\partial U$. A stronger property holds. Let $\psi^{(1)}, \psi^{(2)}$ be two smooth initial conditions for (3.13) with

$$
\psi^{(1)}(u) \geq \psi^{(2)}(u) \text { for every } u \in U \text { and }\left.\psi^{(1)}\right|_{\partial U}=\left.\psi^{(2)}\right|_{\partial U}=\left.\psi_{0}\right|_{\partial U} .
$$

Then, one can show (see end of Section 3.2.2) that

$$
\int_{\partial U}\left[W\left(\nabla \psi^{(1)}\right)-W\left(\nabla \psi^{(2)}\right)\right] \cdot n d \gamma \leq 0
$$

Inequality $\psi^{(1)}(u, t) \geq \psi^{(2)}(u, t)$ remains true for all times, by the usual comparison principle for parabolic PDEs [17, Ch. 3] (another way to convince oneself that order is preserved is to recall that the microscopic dynamics is monotone [20, 3]: if two initial conditions $\eta, \eta^{\prime}$ satisfy $h_{\eta} \leq h_{\eta^{\prime}}$ everywhere, then the two evolutions can be coupled in a way that domination is preserved at all times). One concludes that

$$
\frac{d}{d t}\left(\mathcal{V}\left(\psi^{(1)}(\cdot, t)\right)-\mathcal{V}\left(\psi^{(2)}(\cdot, t)\right)\right)=\int_{\partial U}\left[W\left(\nabla \psi^{(1)}(u, t)\right)-W\left(\nabla \psi^{(2)}(u, t)\right)\right] \cdot n d \gamma \leq 0(3
$$


the drift of mutual volume is a boundary effect and is negative. This fact has a microscopic analog: in fact, if $\eta^{(1)}, \eta^{(2)}$ are two configurations in $\Omega_{U_{L}}$ with $h_{\eta^{(1)}} \geq h_{\eta^{(2)}}$ everywhere in $U_{L}$, then the volume drift

$$
\left[\mathcal{L} \sum_{u \in U_{L}} h(u)\right]\left(\eta^{(1)}\right)-\left[\mathcal{L} \sum_{u \in U_{L}} h(u)\right]\left(\eta^{(2)}\right)
$$

(with $\mathcal{L}$ the generator of the Markov chain) is negative and is non-zero only due to a boundary effect. This was proven in 14 for dynamics I and in 2 for dynamics II, and is actually the crucial step in the proof that the mixing time is polynomial in $L$. In this perspective, it is natural to recover such volume decrease property in the hydrodynamic equation.

It is worth emphasizing that volume contraction is not an a-priori obvious property. In particular, it is easy to check that the single-flip dynamics, at the microscopic level, does not contract volume.

3.1.2. $\mathbb{L}^{2}$ contraction. Let again $\psi^{(1)}(u, t), \psi^{(2)}(u, t)$ be two smooth solutions of (3.3), with the same boundary data on $\partial U$. This time we do not require that $\psi^{(1)} \geq \psi^{(2)}$. We have

$$
\begin{aligned}
& \frac{d}{d t} \int_{U}\left(\psi^{(1)}(u, t)-\psi^{(2)}(u, t)\right)^{2} d u \\
& \quad=-2 \int_{U}\left(\nabla \psi^{(1)}(u, t)-\nabla \psi^{(2)}(u, t)\right) \cdot\left(W\left(\nabla \psi^{(1)}(u, t)\right)-W\left(\nabla \psi^{(2)}(u, t)\right)\right) d u
\end{aligned}
$$

We claim now that

$$
(a-b) \cdot(W(a)-W(b)) \geq 0
$$

whenever $a, b$ belong to the triangle $\mathbb{T}$, which implies that the time derivative in (3.19) is negative. To prove (3.20), it is sufficient to prove that the matrix

$$
H_{W}(\rho):=\left(\begin{array}{cc}
\partial_{\rho_{1}} W^{(1)}(\rho) & \partial_{\rho_{2}} W^{(1)}(\rho) \\
\partial_{\rho_{1}} W^{(2)}(\rho) & \partial_{\rho_{2}} W^{(2)}(\rho)
\end{array}\right)
$$

is positive definite for every $\rho \in \mathbb{T}$. The trace of $H_{W}$ is

$$
\operatorname{Tr}\left(H_{W}(\rho)\right)=\frac{1}{2} \frac{\sin \left(\pi \rho_{1}\right)^{2}+\sin \left(\pi \rho_{2}\right)^{2}}{\sin \left(\pi\left(\rho_{1}+\rho_{2}\right)\right)^{2}}>0
$$

(recall that $\left.\rho_{1}, \rho_{2} \in(0,1)\right)$ while

$$
\begin{aligned}
\operatorname{det}\left(H_{W}(\rho)\right)=\frac{1}{64 \sin \left(\pi\left(\rho_{1}+\rho_{2}\right)\right)^{3}}\{ & 5 \sin \left[\pi\left(\rho_{1}+\rho_{2}\right)\right]+\sin \left[3 \pi\left(\rho_{1}+\rho_{2}\right)\right] \\
& \left.-2 \sin \left[\pi\left(3 \rho_{1}+\rho_{2}\right)\right]-2 \sin \left[\pi\left(\rho_{1}+3 \rho_{2}\right)\right]\right\} .
\end{aligned}
$$

Given that $0<\rho_{1}+\rho_{2}<1$ for $\rho \in \mathbb{T}$, the ratio in the r.h.s. of $(3.23)$ is positive. As for the term $\{\ldots\}$, one can check that its unique extremum for $\rho \in \mathbb{T}$ is at $\rho=$ $(\arctan (\sqrt{5}) / \pi, \arctan (\sqrt{5}) / \pi)$, where $\{\ldots\}>0$.

Remark 12. If the interface mobility $\mu(\cdot)$ were a constant (i.e. if $W$ were proportional to $\nabla \sigma$, in which case (3.3) would be the gradient flow w.r.t. the surface tension functional) then $\mathbb{L}^{2}$ contraction would be an immediate consequence of convexity of the surface 
tension, since the matrix $H_{W}(\rho)$ would be replaced by the Hessian of $\sigma(\sigma)$ computed at $\rho$.

3.2. Another form for the hydrodynamic equation. There exists another way of guessing the hydrodynamic limit equation, this time not based on linear response but on a "local equilibrium" assumption. For this derivation, it is actually more convenient to use a different way of parametrizing the interface and the height function. The corresponding expression for the hydrodynamic limit equation will show an interesting link between mobility and surface tension, see (3.47).

3.2.1. Level set function. Let $P_{110}$ be the linear subspace of $\mathbb{R}^{3}$ orthogonal to $(1,1,0)$ (i.e. the plane $x+y=0)$. On $P_{110}$ we take coordinates $v=\left(v_{1}, v_{2}\right)$ whose unit vectors $\hat{e}_{1}, \hat{e}_{2}$ are the $P_{110}$ orthogonal projections of the Cartesian unit vectors $e_{y}, e_{z}$ of $\mathbb{R}^{3}$ (i.e. $\left.\hat{e}_{1}=\Pi_{110}\left(e_{y}\right), \hat{e}_{2}=\Pi_{110}\left(e_{z}\right)\right)$ and such that the point of coordinates $v=(0,0)$ is the $P_{110}$ projection of $(0,0,0)$. Given $v=\left(v_{1}, v_{2}\right) \in \mathbb{Z} \times(\mathbb{Z}+1 / 2)$, let

$$
\hat{h}(v):=-p \cdot\left(e_{x}+e_{y}\right),
$$

with the usual scalar product on $\mathbb{R}^{3}$ and $p \in \mathbb{Z}^{3}$ the uniquen point of the surface $\Sigma$ whose $\Pi_{110}$ projection is $\left(v_{1}, v_{2}\right)$. We can easily extend $\hat{h}$ to a function on $\mathbb{R}^{2}$ : formula (3.24) is well defined whenever $v_{2} \notin \mathbb{Z}$, and for $v_{2} \in \mathbb{Z}$ (in which case the point $p$ may be not uniquely defined) we let, say, $\hat{h}(v)=\lim _{\epsilon \searrow_{0}} \hat{h}\left(v_{1}, v_{2}-\epsilon\right)$. This choice is somewhat arbitrary but this should be irrelevant in the $L \rightarrow \infty$ limit. As in the case of the height function $\{h(u)\}_{u \in \mathcal{T}}$, the function $\{\hat{h}(v)\}_{v \in \mathbb{Z} \times(\mathbb{Z}+1 / 2)}$ uniquely determines the surface $\Sigma$.

Remark 13. Note that when a particle moves one step up/down, the height function $h$ changes by $-1 /+1$ at some vertex $u \in \mathcal{T}$, while the function $\hat{h}$ changes by $-2 /+2$ at some $v \in \mathbb{Z} \times(\mathbb{Z}+1 / 2)$.

We will call the function $v \mapsto \hat{h}(v)$ the "level set function" of the interface $\Sigma$, in order to distinguish it from the "height function" $u \mapsto h(u)$. The reason for the name is the following. Given $v_{2} \in \mathbb{Z}+1 / 2$, consider the intersection $S^{\left(v_{2}\right)}$ of the surface $\Sigma$ with the horizontal plane $\left\{(x, y, z) \in \mathbb{R}^{3}: z=v_{2}\right\}$. With reference to Figure 7, each $S^{\left(v_{2}\right)}$ can be viewed as a simple-random walk path $\left\{S^{\left(v_{2}\right)}\left(v_{1}\right)\right\}_{v_{1} \in \mathbb{Z}}$ in space-time dimension $(1+1)$ : the time axis $v_{1}$ is horizontal, $S^{\left(v_{2}\right)}\left(v_{1}\right) \in \mathbb{Z}$ and $S^{\left(v_{2}\right)}\left(v_{1}\right)-S^{\left(v_{2}\right)}\left(v_{1}+1\right) \in\{-1,+1\}$. Moreover, these lines are mutually non-intersecting: $S^{\left(v_{2}+1\right)}\left(v_{1}\right) \geq S^{\left(v_{2}\right)}\left(v_{1}\right)$. It is easy to check that, modulo a global additive constant independent of $v=\left(v_{1}, v_{2}\right)$, one has

$$
\hat{h}(v)=S^{\left(v_{2}\right)}\left(v_{1}\right)
$$

Next, we define the analogue (in this new parametrization of the surface) of the domain $U_{L} \subset \mathcal{T}$. Given $U_{L}$ as in Section 2.1 and $\eta \in \Omega_{U_{L}}$, let $\Sigma_{L}$ be the monotone surface whose $\Pi_{111}$ projection is $\eta$. Let $\eta^{\prime}$ and $\Sigma^{\prime}$ denote an arbitrary extension of $\eta$ to a tiling of the whole plane $\mathcal{T}$ and the corresponding monotone surface (see discussion just before Assumption 3). We let

$$
V_{L}:=\Pi_{110}\left(\Sigma_{L}\right)
$$

\footnotetext{
${ }^{1}$ The reason for the choice $v_{2} \in \mathbb{Z}+1 / 2$ is that if instead we required that $v_{2} \in \mathbb{Z}$, then $p$ would not be uniquely defined (horizontal square faces of $\Sigma$ are projected into segments at some integer vertical height $v_{2}$ ).
} 


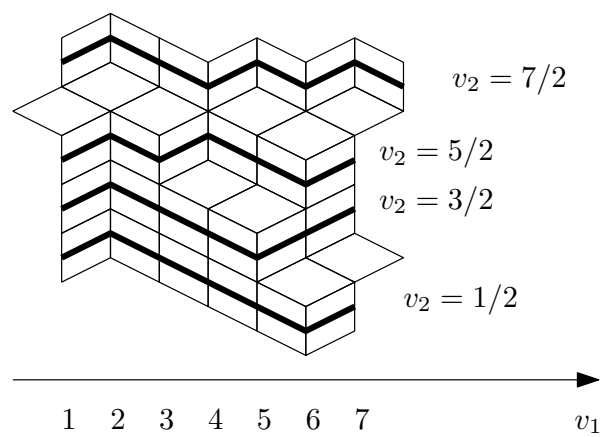

FiguRE 7.

and we note that $V_{L}$ is independent of the choice of $\eta \in \Omega_{U_{L}}$. Actually, on $\mathbb{R}^{2} \backslash V_{L}$ the function $v \mapsto \hat{h}(v)$ depends only on the arbitrary choice of $\eta^{\prime}$ outside of $U_{L}$.

The following is equivalent to Assumption 3 and is actually a rephrasing of it:

Proposition 14. As $L \rightarrow \infty,(1 / L) V_{L}$ tends in Hausdorff distance to a bounded simply connected closed domain $V \subset \mathbb{R}^{2}$ with smooth boundary. There is a continuous function $\hat{\phi}$ on $\partial V$ and if $v^{(L)} \in \mathbb{R}^{2} \backslash V_{L}$ is such that $v^{(L)} / L \rightarrow v \in \partial V$ as $L \rightarrow \infty$ then

$$
\frac{1}{L} \hat{h}\left(v^{(L)}\right) \rightarrow \hat{\phi}(v)
$$

Moreover, there exists a $C^{1}$ function $\hat{\psi}: V \mapsto \mathbb{R}$ such that $\hat{\psi}=\hat{\phi}$ on $\partial V$ and

$$
\nabla \hat{\psi}(v) \in(-1,1) \times(0, \infty) \quad \text { for every } \quad v \in V .
$$

All these claims follow from Assumption 3 and the change of variable formulas in Section 5.1 below; in particular, Eq. (5.4) shows that (3.28) is equivalent to the nonextremality condition 2.1.

3.2.2. Hydrodynamic limit for the level set function. Let for $v \in V$ and $t \geq 0$

$$
\hat{H}(v, t)=\frac{1}{L} \hat{h}_{\eta\left(t L^{2}\right)}(v L) \text {. }
$$

Recall that we assume that the initial condition of the dynamics satisfies (3.1). As in Proposition 14, in terms of the "level set function" this implies that there exists a smooth $\hat{\psi}_{0}: v \in V \mapsto \psi_{0}(v) \in \mathbb{R}$ satisfying (3.28) such that

$$
\lim _{L \rightarrow \infty} \hat{H}(v, 0)=\hat{\psi}_{0}(v), \quad \forall v \in V .
$$

The conjectural existence of a hydrodynamic limit means existence of a function $\psi(\cdot, \cdot)$ : $V \times \mathbb{R}^{+} \mapsto \mathbb{R}$, such that for every $\epsilon>0$,

$$
\mathbb{P}(\exists v \in V:|\hat{H}(v, t)-\hat{\psi}(v, t)| \geq \epsilon) \stackrel{L \rightarrow \infty}{\rightarrow} 0 .
$$

Under a (reasonable) assumption of local equilibrium, we find (see Section 5.2 that $\hat{\psi}$ has to satisfy the PDE

$$
\left\{\begin{array}{l}
\partial_{t} \hat{\psi}=\frac{1}{2} \partial_{v_{1}}^{2} \hat{\psi}-\frac{1}{4} \partial_{v_{2}}\left[\left(2+\partial_{v_{2}} \hat{\psi}\right) \pi_{\rho(\nabla \hat{\psi})}\left[\left(\left|n\left(b^{(0,0)}\right)\right|-1\right) 1_{X(0,0)} 1_{n\left(b^{(0,0)}\right) \neq 0}\right]\right] \\
\hat{\psi}(v, t)=\hat{\psi}_{0}(v) \quad \text { if } t=0 \text { or if } v \in \partial V
\end{array}\right.
$$


where $\rho(\nabla \hat{\psi})=\left(\rho_{1}(\nabla \hat{\psi}), \rho_{2}(\nabla \hat{\psi})\right)$ is defined as

$$
\rho_{1}\left(s_{1}, s_{2}\right)=\frac{1-s_{1}}{2+s_{2}}, \quad \rho_{2}\left(s_{1}, s_{2}\right)=\frac{1+s_{1}}{2+s_{2}} .
$$

We will prove in Section 6 :

Proposition 15. The following identities hold:

$$
\pi_{\rho}\left(1_{X(0,0)} 1_{n\left(b^{(0,0)}\right) \neq 0}\right)=2\left[\rho_{1} \rho_{2}+\left(1-\rho_{1}-\rho_{2}\right) V(\rho)\right]
$$

and

$$
\pi_{\rho}\left[\left(\left|n\left(b^{(0,0)}\right)\right|-1\right) 1_{X(0,0)} 1_{n\left(b^{(0,0)}\right) \neq 0}\right]=2\left[-\rho_{1} \rho_{2}+\left(\rho_{1}+\rho_{2}\right) V(\rho)\right] .
$$

Equation (3.32) then becomes

$$
\partial_{t} \hat{\psi}=\frac{1}{2} \partial_{v_{1}}^{2} \hat{\psi}+\left.\left[\partial_{s_{1}} G(s) \partial_{v_{1} v_{2}}^{2} \hat{\psi}+\partial_{s_{2}} G(s) \partial_{v_{2}}^{2} \hat{\psi}\right]\right|_{s=\left(s_{1}, s_{2}\right)=\nabla \hat{\psi}}
$$

where

$$
G(s)=\left(1+s_{2} / 2\right)\left[\rho_{1}\left(s_{1}, s_{2}\right) \rho_{2}\left(s_{1}, s_{2}\right)-\left(\rho_{1}\left(s_{1}, s_{2}\right)+\rho_{2}\left(s_{1}, s_{2}\right)\right) V\left(\rho\left(s_{1}, s_{2}\right)\right)\right]
$$

and $\rho(\cdot, \cdot)$ is defined in $(3.33)$.

The derivatives of $G$ are a combination of trigonometric functions, but it is best to express them in terms of the surface tension. Given Theorem 4 , it is easy to deduce (see Section 5.1) that, given $\hat{\psi}: V \mapsto \mathbb{R}$ satisfying (3.28), one has

$$
\lim _{\delta \rightarrow 0} \lim _{L \rightarrow \infty} \ln \left|\left\{\eta \in \Omega_{U_{L}}: \sup _{v \in V}\left|L^{-1} \hat{h}_{\eta}(v L)-\hat{\psi}(v)\right| \leq \delta\right\}\right|=-\int_{V} \hat{\sigma}(\nabla \hat{\psi}) d v
$$

where

$$
\hat{\sigma}(s)=\hat{\sigma}\left(s_{1}, s_{2}\right)=\left(1+\frac{s_{2}}{2}\right) \sigma\left(\rho\left(s_{1}, s_{2}\right)\right) .
$$

Then, one can check ${ }^{2}$ from $(3.39)$ and $(3.37)$ that the following simple relations hold:

$$
\partial_{s_{1}} G(s)=\frac{\hat{\sigma}_{1,2}(s)}{\hat{\sigma}_{1,1}(s)}, \quad \partial_{s_{2}} G(s)=\frac{1}{2} \frac{\hat{\sigma}_{2,2}(s)}{\hat{\sigma}_{1,1}(s)}
$$

with $\hat{\sigma}_{i, j}:=\partial^{2} \hat{\sigma} / \partial s_{i} \partial s_{j}$. Altogether, the conjectural hydrodynamic limit equation is

$$
\left\{\begin{array}{l}
\partial_{t} \hat{\psi}=\frac{1}{2} \partial_{v_{1}}^{2} \hat{\psi}+\frac{\hat{\sigma}_{1,2}(\nabla \hat{\psi})}{\hat{\sigma}_{1,1}(\nabla \hat{\psi})} \partial_{v_{1} v_{2}}^{2} \hat{\psi}+\frac{1}{2} \frac{\hat{\sigma}_{2,2}(\nabla \hat{\psi})}{\hat{\sigma}_{1,1}(\nabla \hat{\psi})} \partial_{v_{2}}^{2} \hat{\psi} \\
\hat{\psi}(v, t)=\hat{\psi}_{0}(v) \quad \text { if } t=0 \text { or if } v \in \partial V .
\end{array}\right.
$$

Remark 16. From 3.40 it follows that the vector field

$$
s \in(-1,1) \times \mathbb{R}^{+} \mapsto\left(\frac{\hat{\sigma}_{1,2}(s)}{\hat{\sigma}_{1,1}(s)}, \frac{1}{2} \frac{\hat{\sigma}_{2,2}(s)}{\hat{\sigma}_{1,1}(s)}\right)
$$

\footnotetext{
${ }^{2}$ The right- and left-hand sides of 3.40 are rather complicated trigonometric functions, and we found no better way to check the equalities than with the help of symbolic computation using Mathematica.
} 
has zero curl, which of course could also be checked directly from the definition of $\hat{\sigma}$. This is reminiscent of a surprising identity discovered in [9]. Namely, one easily deduces from (3.7) that

$$
\operatorname{det}\left(\begin{array}{ll}
\sigma_{1,1} & \sigma_{1,2} \\
\sigma_{1,2} & \sigma_{2,2}
\end{array}\right)=\pi^{2} .
$$

(Actually, identity (3.43) holds more generally for the dimer model on any infinite, translation invariant, planar bipartite lattice [9, Sec. 5.3.3] and it is deduced from algebraic properties of so-called spectral curves [9, Sec. 3.2.3] of the dimer model.) Taking the derivative of (3.43) w.r.t. $\rho_{1}$ one obtains

$$
\sigma_{1,1,1} \sigma_{2,2}+\sigma_{1,1} \sigma_{1,2,2}=2 \sigma_{1,2} \sigma_{1,1,2}
$$

with of course $\sigma_{a, b, c}=\partial_{\rho_{a}} \partial_{\rho_{b}} \partial_{\rho_{c}} \sigma(\rho)$. In turn, this is immediately seen to be equivalent to the vanishing of the curl of the vector field

$$
\rho \in \mathbb{T} \mapsto\left(\frac{\sigma_{1,2}(\rho)}{\sigma_{1,1}(\rho)}, \frac{1}{2} \frac{\sigma_{2,2}(\rho)}{\sigma_{1,1}(\rho)}\right) .
$$

Equation (3.45) is strikingly similar to (3.42) but it is not obvious how to relate via simple algebra the vanishing of the curl of the former with that of the latter. The probabilistic meaning and the relation between these two identities deserves to be further explored.

For more general random surface models, there is no reason to expect that the l.h.s. of (3.43) is independent of the slope (convexity of the surface tension says just that the determinant is positive) but in [1] it was argued that (3.43) should hold at slopes corresponding to cusps of $\sigma$.

A couple of comments are in order. First, as it should, equations (3.41) and (3.3) (together with (3.12) ) are actually the same PDE, as can be seen with a suitable change of variables (see Section 5.1 for details). A second remark is that in the $\left(v_{1}, v_{2}\right)$ coordinate system one obtains a surprisingly simple relation between interface mobility and surface tension. Namely, rewriting (in analogy with (3.3) ) the PDE satisfied by $\hat{\psi}$ as

$$
\partial_{t} \hat{\psi}=\hat{\mu}(\nabla \hat{\psi}) \sum_{i, j=1,2} \hat{\sigma}_{i, j}(\nabla \hat{\psi}) \frac{\partial^{2}}{\partial_{v_{i}} \partial_{v_{j}}} \hat{\psi}
$$

and comparing with (3.41) one sees that the mobility coefficient is

$$
\hat{\mu}(\nabla \hat{\psi})=\frac{1}{2 \hat{\sigma}_{1,1}(\nabla \hat{\psi})} .
$$

It would be interesting to understand whether there is any thermodynamic explanation for such relation.

Finally, from 3.36 one sees that the equation for $\hat{\psi}$ is also given by

$$
\partial_{t} \hat{\psi}=\operatorname{div}\left((1 / 2) \partial_{v_{1}} \hat{\psi}, G(\nabla \hat{\psi})\right),
$$

from which one finds again that the time derivative of the total volume is a boundary term:

$$
\frac{d}{d t} \int_{V} \hat{\psi}(v, t)=\int_{\partial V}\left((1 / 2) \partial_{v_{1}} \hat{\psi}, G(\nabla \hat{\psi})\right) \cdot n d \gamma
$$


Take now two initial conditions with $\hat{\psi}^{(1)}(v) \geq \hat{\psi}^{(2)}(v)$ for every $v \in V$ (or equivalently $\psi^{(1)}(u) \geq \psi^{(2)}(u)$ for every $\left.u \in U\right)$ and coinciding on $\partial V$. We want to show that

$$
\frac{d}{d t} \int_{V}\left(\hat{\psi}^{(1)}(v, t)-\hat{\psi}^{(2)}(v, t)\right) d v \leq 0 .
$$

As we already observed after $(3.16)$, the property $\psi^{(1)} \geq \psi^{(2)}$ is preserved at later times, by the comparison principle for parabolic PDEs. One deduces

$$
\begin{array}{r}
\frac{d}{d t} \int_{V}\left(\hat{\psi}^{(1)}-\hat{\psi}^{(2)}\right) d v=\int_{\partial V}\left((1 / 2) \partial_{v_{1}}\left(\hat{\psi}^{(1)}-\hat{\psi}^{(2)}\right), G\left(\nabla \hat{\psi}^{(1)}\right)-G\left(\nabla \hat{\psi}^{(2)}\right)\right) \cdot n d \gamma \\
=\frac{1}{2} \int_{\partial V} n_{1} \partial_{v_{1}}\left(\hat{\psi}^{(1)}-\hat{\psi}^{(2)}\right) d \gamma+\int_{\partial V} n_{2}\left(G\left(\nabla \hat{\psi}^{(1)}\right)-G\left(\nabla \hat{\psi}^{(2)}\right)\right) d \gamma
\end{array}
$$

with $n_{i}$ the $i^{\text {th }}$ component of the exterior normal vector $n$. Since $\hat{\psi}^{(1)} \geq \hat{\psi}^{(2)}$ and they coincide on $\partial V$, for $v_{0} \in \partial V$ we have that ${ }^{3}$

$$
\operatorname{sign}\left[\lim _{v \rightarrow v_{0}} \partial_{v_{i}}\left(\hat{\psi}^{(1)}-\hat{\psi}^{(2)}\right)\right]=-\operatorname{sign}\left[n_{i}\left(v_{0}\right)\right], \quad i=1,2,
$$

whenever $n_{i}\left(v_{0}\right) \neq 0$ (here, $n\left(v_{0}\right)$ is the exterior normal at $v_{0}$ ). To show (3.50) (and actually that the integrand of (3.51) itself is point-wise negative) it suffices to show that

$$
\operatorname{sign}\left[\lim _{v \rightarrow v_{0}} \partial_{v_{2}}\left(\hat{\psi}^{(1)}-\hat{\psi}^{(2)}\right)\right]=\operatorname{sign}\left[\lim _{v \rightarrow v_{0}}\left(G\left(\nabla \hat{\psi}^{(1)}\right)-G\left(\nabla \hat{\psi}^{(2)}\right)\right)\right]
$$

whenever $n_{2}\left(v_{0}\right) \neq 0$. Take the limit $v \rightarrow v_{0}$ in direction $v_{2}$. Then, the claim follows if we know that $G\left(s_{1}, s_{2}\right)$ is increasing in its second argument. In turn, this follows from the second of $(3.40)$ plus convexity of the surface tension $\hat{\sigma}$.

From formulas (5.12) and (5.3) of Section 5.1 one sees that

$$
\frac{d}{d t} \int_{U} \psi(u, t) d u=\frac{1}{2} \frac{d}{d t} \int_{V} \hat{\psi}(v, t) d v
$$

Then, (3.16) follows.

\section{Mobility and Linear Response theory}

Let us briefly recall the method of [16] for the computation of the mobility. The idea is to consider the dynamics in a finite volume $\Lambda$ of diameter $O(L)$ and to modify the rates through a "magnetic field" $B>0$ such that the invariant measure $\pi_{\Lambda}^{(B)}$ is tilted by the exponential of $B$ times the volume (the volume being the sum of the heights in $\Lambda$ ) w.r.t. the $B=0$ situation. In presence of magnetic field, the free energy (3.5) becomes $F(\psi)=\int_{U} \sigma(\nabla \psi) d u-B \int_{U} \psi d u$, where the surface tension $\sigma$ is as in Theorem 4. The transition rates are changed in an easy way by $B$ : If for $B=0$ the rate at which the

\footnotetext{
${ }^{3}$ Recall that we are assuming that $\hat{\psi}(\cdot, t)$ is $C^{1}$ in $V$ for all times, see discussion at the beginning of Section 3.1

${ }^{4}$ The factor $1 / 2$ in Eq. 3.54 can be intuitively understood from the microscopic dynamics, recalling Remark 13
} 
vertical position of particle $b$ changes from $n(b)$ (the present position in $\eta$ ) to $n(b)+y$, $y \in \mathbb{Z}, y \neq 0$ is denoted $c_{b, y}(\eta)$, more explicitly (recalling Definitions 7 and 8 )

$$
c_{b, y}^{I}(\eta)=\frac{1}{2|y|} 1_{\{n(b)+y \in I(b)\}}
$$

and

$$
c_{b, y}^{I I}(\eta)=\frac{1}{|I(b)|} 1_{\{n(b)+y \in I(b)\}},
$$

for dynamics I and II respectively, then for $B \neq 0$ one has

$$
c_{b, y}^{B}(\eta)=c_{b, y}(\eta) \exp (B y / 2) .
$$

The heuristics that leads to (3.6) should still apply so the hydrodynamic limit should follow the equation

$$
\begin{aligned}
\partial_{t} \psi=-\mu(\nabla \psi) \frac{\delta}{\delta \psi}\left(\int_{U} \sigma(\nabla \psi) d u\right. & \left.-B \int_{U} \psi d u\right) \\
& =\mu(\nabla \psi) \sum_{i, j=1,2} \sigma_{i, j}(\nabla \psi) \frac{\partial^{2}}{\partial_{u_{i}} \partial_{u_{j}}} \psi+\mu(\nabla \psi) B .
\end{aligned}
$$

If the configuration $\psi$ is flat (i.e. has constant gradient $\rho$ on $U$ ), one has then

$$
\partial_{t} \psi=B \mu(\rho)
$$

so the $B=0$ value of the mobility is obtained (within linear response theory) as the derivative of the interface velocity w.r.t. $B$, at $B=0$. The system is started from the $B=0$ reversible measure $\pi_{\Lambda}$ on $\Lambda$, with boundary conditions chosen such that the law of the gradients is translation-invariant and corresponds to the desired slope $\rho$. Then, the box side $L$ is taken to $+\infty$ at fixed time (so that boundary effects do not prevent the law of the gradients from remaining translation invariant at positive time) and finally time is taken to $+\infty$, in order to ensure that the system has reached its asymptotic velocity. Note that if time were taken to infinity first, then the asymptotic velocity would be 0 since the system would reach its new invariant measure $\pi_{\Lambda}^{(B)}$.

Let us implement this scheme in our case. For technical convenience, we take $\Lambda \subset \mathcal{T}$ to be $L$-periodic both in direction $e_{1}$ and in direction $e_{2}$. Note that any tiling of $\Lambda$ contains $L^{2}$ lozenges. In order to impose the tilt $\rho$, we let $\pi_{\Lambda}:=\pi_{\Lambda, \rho^{(L)}}$ denote the uniform measure on lozenge tilings of $\Lambda$ such that the fraction of north-west (resp. north-east) oriented lozenges is $\rho_{1}^{(L)}$ (resp. $\rho_{2}^{(L)}$ ), with $\rho_{i}^{(L)} \rightarrow \rho_{i}$ as $L \rightarrow \infty$. It is then known [9] that $\pi_{\Lambda}$ converges to $\pi_{\rho}$, in the sense that averages of local functions converge.

The drift of height per unit site (i.e. the interface velocity) at time $t$ is

$$
\frac{1}{L^{2}} \sum_{b, y} \mathbb{E}_{\pi_{\Lambda}}\left[c_{b, y}^{B}(\eta(t)) y\right]=\frac{1}{L^{2}} \sum_{\eta, \eta^{\prime}} \sum_{b, y} \pi_{\Lambda}(\eta) e^{t \mathcal{L}^{B}}\left(\eta, \eta^{\prime}\right) c_{b, y}^{B}\left(\eta^{\prime}\right) y
$$

with the average on the law of the process at time $t$ started from the $\pi_{\Lambda}$ and the sum over $b$ running over particles in $\Lambda$ (there are exactly $L^{2}\left(1-\rho_{1}^{(L)}-\rho_{2}^{(L)}\right)$ of them). Here $\mathcal{L}^{B}$ is the generator of the process with rates $c_{b, y}^{B}$. 
Recall that we want to take the $B$ derivative of $(4.6)$ at $B=0$, then the limit $L \rightarrow \infty$ and finally the limit $t \rightarrow \infty$ to obtain the mobility $\mu(\rho)$. Taking the derivative and using

$$
\left.\frac{d}{d B} c_{b, y}^{B}(\eta)\right|_{B=0}=c_{b, y}(\eta) \frac{y}{2}
$$

together with invariance of $\pi_{\Lambda}$ for the $B=0$ process, one gets

$$
\frac{1}{2} \frac{1}{L^{2}} \sum_{b, y} \sum_{\eta} \pi_{\Lambda}(\eta) c_{b, y}(\eta) y^{2}+\frac{1}{L^{2}} \sum_{\eta, b, y, \eta^{\prime}, \eta^{\prime \prime}} \int_{0}^{t} \pi_{\Lambda}(\eta) \mathcal{L}^{\prime}\left(\eta, \eta^{\prime}\right) e^{\tau \mathcal{L}}\left(\eta^{\prime}, \eta^{\prime \prime}\right) c_{b, y}\left(\eta^{\prime \prime}\right) y d \tau
$$

where $\mathcal{L}^{\prime}\left(\eta, \eta^{\prime}\right)=\left.\partial_{B} \mathcal{L}\left(\eta, \eta^{\prime}\right)\right|_{B=0}$. We have

$$
\begin{gathered}
\sum_{\eta, \eta^{\prime}, \eta^{\prime \prime}} \pi_{\Lambda}(\eta) \mathcal{L}^{\prime}\left(\eta, \eta^{\prime}\right) e^{\tau \mathcal{L}}\left(\eta^{\prime}, \eta^{\prime \prime}\right) c_{b, y}\left(\eta^{\prime \prime}\right) y \\
=\sum_{\eta, \eta^{\prime}} \pi_{\Lambda}(\eta) \mathcal{L}^{\prime}(\eta, \eta) e^{\tau \mathcal{L}}\left(\eta, \eta^{\prime}\right) c_{b, y}\left(\eta^{\prime}\right) y+\sum_{\eta, \eta^{\prime}, \eta^{\prime \prime}: \eta^{\prime} \neq \eta} \pi_{\Lambda}(\eta) \mathcal{L}^{\prime}\left(\eta, \eta^{\prime}\right) e^{\tau \mathcal{L}}\left(\eta^{\prime}, \eta^{\prime \prime}\right) c_{b, y}\left(\eta^{\prime \prime}\right) y .
\end{gathered}
$$

Note that $\mathcal{L}^{\prime}\left(\eta, \eta^{\prime}\right.$ ) (with $\eta \neq \eta^{\prime}$ ) is non-zero only if one can go from $\eta$ to $\eta^{\prime}$ in a single move (say $\left.b^{\prime}, z\right)$ and in that case $\mathcal{L}^{\prime}\left(\eta, \eta^{\prime}\right)=c_{b^{\prime}, z}(\eta) \frac{z}{2}$. Then, the last sum becomes

$$
\sum_{\eta, \eta^{\prime \prime}, b^{\prime}, z} \pi_{\Lambda}(\eta) c_{b^{\prime}, z}(\eta) \frac{z}{2} e^{\tau \mathcal{L}}\left(\eta^{b^{\prime}, z}, \eta^{\prime \prime}\right) c_{b, y}\left(\eta^{\prime \prime}\right) y
$$

with $\eta^{b^{\prime}, z}$ the configuration where $b^{\prime}$ has been moved by $z$. Changing names of variables this is also equal to

$$
\begin{aligned}
& \sum_{b^{\prime}, z} \sum_{\eta, \eta^{\prime}} \pi_{\Lambda}\left(\eta^{b^{\prime},-z}\right) c_{b^{\prime}, z}\left(\eta^{b^{\prime},-z}\right) \frac{z}{2} e^{\tau \mathcal{L}}\left(\eta, \eta^{\prime}\right) c_{b, y}\left(\eta^{\prime}\right) y \\
= & -\frac{1}{2} \sum_{b^{\prime}, z} \sum_{\eta, \eta^{\prime}} \pi_{\Lambda}\left(\eta^{b^{\prime}, z}\right) c_{b^{\prime},-z}\left(\eta^{b^{\prime}, z}\right) z e^{\tau \mathcal{L}}\left(\eta, \eta^{\prime}\right) c_{b, y}\left(\eta^{\prime}\right) y .
\end{aligned}
$$

Using detailed balance, $\pi_{\Lambda}\left(\eta^{b^{\prime}, z}\right) c_{b^{\prime},-z}\left(\eta^{b^{\prime}, z}\right)=\pi_{\Lambda}(\eta) c_{b^{\prime}, z}(\eta)$. Therefore 4.10 is equal to

$$
\sum_{\eta, \eta^{\prime}} \pi_{\Lambda}(\eta)\left[\mathcal{L}^{\prime}(\eta, \eta)-\sum_{\eta^{\prime \prime}: \eta^{\prime \prime} \neq \eta} \mathcal{L}^{\prime}\left(\eta, \eta^{\prime \prime}\right)\right] e^{\tau \mathcal{L}}\left(\eta, \eta^{\prime}\right) c_{b, y}\left(\eta^{\prime}\right) y .
$$

Since $\sum_{\eta^{\prime \prime}} \mathcal{L}\left(\eta, \eta^{\prime \prime}\right)=0$, the same is true for $\mathcal{L}^{\prime}$ and (4.14) gives

$$
-2 \sum_{\eta, \eta^{\prime}, \eta^{\prime \prime}: \eta^{\prime \prime} \neq \eta} \pi_{\Lambda}(\eta) \mathcal{L}^{\prime}\left(\eta, \eta^{\prime \prime}\right) e^{\tau \mathcal{L}}\left(\eta, \eta^{\prime}\right) c_{b, y}\left(\eta^{\prime}\right) y .
$$

Altogether, using also 4.7), 4.8 gives

$$
\frac{1}{2 L^{2}} \pi_{\Lambda}\left[\sum_{b, y} c_{b, y}(\eta) y^{2}\right]-\frac{1}{L^{2}} \sum_{b, y, b^{\prime}, z} \int_{0}^{t} \mathbb{P}_{\pi_{\Lambda}}\left[c_{b^{\prime}, z}(\eta(0)) z c_{b, y}(\eta(\tau)) y\right] d \tau .
$$


Finally, letting first $L \rightarrow \infty$ and then $t \rightarrow \infty$, the mobility provided by the linear response theory is

$$
\mu(\rho)=\lim _{L \rightarrow \infty} \frac{1}{2 L^{2}} \pi_{\Lambda}\left[\sum_{b, y} c_{b, y}(\eta) y^{2}\right]-\int_{0}^{\infty} \lim _{L \rightarrow \infty} \frac{1}{L^{2}} \sum_{b, y, b^{\prime}, z} \mathbb{P}_{\pi_{\Lambda}}\left[c_{b^{\prime}, z}(\eta(0)) z c_{b, y}(\eta(\tau)) y\right] d \tau .
$$

A simple computation shows that, both for dynamics I and II,

$$
\pi_{\Lambda}\left[\sum_{y} c_{b, y}(\eta) y^{2} \mid n^{+}(b), n^{-}(b)\right]=\frac{1}{6}\left(|I(b)|^{2}-1\right)=\frac{1}{6}(|I(b)|+1)(|I(b)|-1),
$$

where we recall that $n^{+}(b), n^{-}(b)$ are the highest/lowest vertical position particle $b$ can take, given the positions of the others, and $I(b)=\left\{n^{-}(b), \ldots, n^{+}(b)\right\}$. Let us see why. The point is that, under the uniform measure $\pi_{\Lambda}$ and conditionally on $n^{ \pm}(b)$, the position $n(b)$ of particle $b$ is uniform in $I(b)$. Then, for dynamics I we have

$$
\begin{aligned}
\pi_{\Lambda}\left[\sum_{y} c_{b, y}(\eta) y^{2} \mid n^{+}(b), n^{-}(b)\right] & =\frac{1}{2} \pi_{\Lambda}\left[\sum_{y: n^{-}(b) \leq n(b)+y \leq n^{+}(b)}|y| n^{+}(b), n^{-}(b)\right] \\
& =\frac{1}{2}|I(b)| \mathbb{E}|U-V|,
\end{aligned}
$$

with $U, V$ two independent random variables, uniformly distributed in $I(b)$. An immediate calculation shows that

$$
\mathbb{E}|U-V|=\frac{1}{3} \frac{|I(b)|^{2}-1}{|I(b)|}
$$

and (4.18) follows. For dynamics II one has instead

$$
\begin{aligned}
& \pi_{\Lambda}\left[\sum_{y} c_{b, y}(\eta) y^{2} \mid n^{+}(b), n^{-}(b)\right]=\frac{1}{|I(b)|} \pi_{\Lambda}\left[\sum_{y: n^{-}(b) \leq n(b)+y \leq n^{+}(b)} y^{2} \mid n^{+}(b), n^{-}(b)\right] \\
& =\mathbb{E}|U-V|^{2},
\end{aligned}
$$

with $U, V$ uniform on $I(b)$ as before. A simple computation gives

$$
\mathbb{E}|U-V|^{2}=\frac{1}{6}\left(|I(b)|^{2}-1\right)
$$

and again 4.18 follows.

Let us resume from Eqs. (4.16) and (4.17) the computation of the mobility. For every particle $b$, there exist exactly $|I(b)|$ sites $u \in \Lambda$ such that $b^{(u)}=b$ (recall that $b^{(u)}$ was defined just before (3.8) ) and exactly one of those sites satisfies $\left(u_{1}+u_{2}\right) / 2=n(b)=$ $n\left(b^{(u)}\right)$. Therefore, the first term in (4.16) is

$$
\frac{1}{12 L^{2}} \pi_{\Lambda}\left[\sum_{u \in \Lambda}\left(\left|I\left(b^{(u)}\right)\right|+1\right) 1_{X(u)} 1_{n\left(b^{(u)}\right) \neq\left(u_{1}+u_{2}\right) / 2}\right],
$$

where the indicator function that $n\left(b^{(u)}\right) \neq\left(u_{1}+u_{2}\right) / 2$ reconstructs the factor $(|I(b)|-1)$ in 4.18 and the indicator $1_{X(u)}$ excludes the sites $u$ for which $b^{(u)}$ is not defined. By 
translation invariance and convergence of $\pi_{\Lambda}$ to $\pi_{\rho}$ as $L \rightarrow \infty$, this converges td 5

$$
\frac{1}{12} \pi_{\rho}\left(\left(\left|I\left(b^{(0,0)}\right)\right|+1\right) 1_{X((0,0))} 1_{n\left(b^{(0,0)}\right) \neq 0}\right) .
$$

Now let us look at the second term in (4.17). Both for dynamics I and II one has, by direct computation,

$$
\sum_{y} c_{b, y}(\eta) y=\frac{1}{2}\left[n^{+}(b)-2 n(b)+n^{-}(b)\right]=\frac{1}{2}\left[\left|I^{+}(b)\right|-\left|I^{-}(b)\right|\right]
$$

where $I^{+}(b)=\{k \in I(b): k>n(b)\}$ and $I^{-}(b)=\{k \in I(b): k<n(b)\}$ (i.e. the set of positions available for $b$ above/below the present position). It was proven in [19, Sec. 4] that

$$
\sum_{b}\left[\left|I^{+}(b)\right|-\left|I^{-}(b)\right|\right]=0
$$

for any configuration. (Here it is important that we are working on the torus $\Lambda$ to avoid boundary terms). Altogether, (3.8) follows.

\section{Derivation of the hydrodynamic EQUation Under ASSUmption of LOCAL EQUILIBRIUM}

5.1. Changes of variables. Given a function $\psi: u \in U \mapsto \psi(u)$ satisfying (2.1), call $\Gamma \subset \mathbb{R}^{3}$ the surface whose height function is $\psi$ : the point $p=(x, y, z) \in \Gamma$ whose $\Pi_{111}$ projection is $u \in U$ (i.e. such that $p=(a, a, a)+\left(u_{1}, u_{2}, 0\right)$ for some $a$ ) has vertical coordinate $z=a=-\psi(u)$. Also let $\hat{\psi}: v \in V \mapsto \hat{\psi}(v)$ be the corresponding level set function: the point $p=(x, y, z) \in \Gamma$ whose $\Pi_{110}$ projection is $v$ (i.e. $p=$ $(b, b, 0)+\left(-v_{1} / 2, v_{1} / 2, v_{2}\right)$ for some $\left.b\right)$ satisfies $-p \cdot\left(e_{x}+e_{y}\right)=-2 b=\hat{\psi}(v)$, see (3.24). Conversely, if $(x, y, z) \in \Gamma$ then its $\left(u_{1}, u_{2}\right)$ and $\left(v_{1}, v_{2}\right)$ coordinates satisfy

$$
u_{1}=x-z, u_{2}=y-z, \psi(u)=-z
$$

and

$$
v_{1}=y-x, v_{2}=z, \hat{\psi}(v)=-x-y .
$$

Given $u \in U$, let $v(u) \in V$ be the $\Pi_{110}$ projection of the unique point $p \in \Gamma$ whose $\Pi_{111}$ projection is $u$. The function $u: v \in V \mapsto u(v)$ will denote the inverse $\epsilon^{6}$ of $v(\cdot)$. By simple geometric considerations (see the end of this subsection), one finds that the area element $d u=d u_{1} d u_{2}$ equals

$$
d u=\frac{d v}{\partial_{u_{1}} \psi(u(v))+\partial_{u_{2}} \psi(u(v))} .
$$

One can check this also with a microscopic argument: a finite portion $\Sigma^{\prime} \subset \Sigma$ composed of $N$ square faces, once projected on $P_{111}$, contains $N$ lozenges and has area $N$ in the

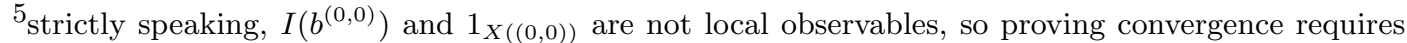
some (doable) technical work. Since anyway linear response theory involves other non-rigorous steps that are much harder to justify, we do not give details on this point.

${ }^{6}$ The function $v(\cdot)$ is bijective thanks to 2.1 : bijectivity could be lost if one allowed $\nabla \psi$ to take the value $(0,0)$, since horizontal regions of $\Gamma$ with normal vector $(0,0,1)$ project into segments of the plane $P_{110}$.
} 
$u_{1}, u_{2}$ coordinates. Assume that $N \rho_{i}$ lozenges are of type $i$. When one projects $\Sigma^{\prime}$ on $P_{110}$ instead, horizontal faces are mapped to zero-area segments and the other faces to area-1 squares. Altogether, the area of the $P_{110}$ projection is $N\left(\rho_{1}+\rho_{2}\right)$ and $(5.3)$ follows from the identification between $\partial_{u_{i}} \psi, i=1,2$ and $\rho_{i}$.

Moreover, one has

$$
\begin{gathered}
\partial_{u_{1}} \psi(u)=\frac{1-\partial_{v_{1}} \hat{\psi}(v(u))}{2+\partial_{v_{2}} \hat{\psi}(v(u))}=\rho_{1}(\nabla \hat{\psi}(v(u))) \\
\partial_{u_{2}} \psi(u)=\frac{1+\partial_{v_{1}} \hat{\psi}(v(u))}{2+\partial_{v_{2}} \hat{\psi}(v(u))}=\rho_{2}(\nabla \hat{\psi}(v(u))),
\end{gathered}
$$

where $\rho(\cdot)$ was already defined in (3.33). To see this, call $n=\left(n_{1}, n_{2}, n_{3}\right)$ the normal vector of the surface $\Gamma$ at some point $p_{0}=\left(x_{0}, y_{0}, z_{0}\right)$, normalized so that $n_{1}+n_{2}+n_{3}=1$. Let us assume w.l.o.g. that $p_{0}=(0,0,0)$. At first order around $p_{0}$, the coordinates of a point $p=(x, y, z) \in \Gamma$ verify

$$
x n_{1}+y n_{2}+z n_{3}=O\left(|p|^{2}\right) .
$$

One then immediately verifies that the height function $\psi(\cdot)$ and the level set function $\hat{\psi}(\cdot)$ verify

$$
\psi(u)=n_{1} u_{1}+n_{2} u_{2}+O\left(|u|^{2}\right)
$$

$$
\hat{\psi}(v)=\frac{\left(n_{2}-n_{1}\right) v_{1}+2 n_{3} v_{2}}{n_{1}+n_{2}}+O\left(|v|^{2}\right),
$$

so that

$$
\begin{aligned}
\partial_{v_{1}} \hat{\psi}(0)=\frac{n_{2}-n_{1}}{n_{1}+n_{2}}=\frac{\partial_{u_{2}} \psi(0)-\partial_{u_{1}} \psi(0)}{\partial_{u_{1}} \psi(0)+\partial_{u_{2}} \psi(0)} \\
\partial_{v_{2}} \hat{\psi}(0)=\frac{2 n_{3}}{n_{1}+n_{2}}=\frac{2\left(1-\partial_{u_{1}} \psi(0)+\partial_{u_{2}} \psi(0)\right)}{\partial_{u_{1}} \psi(0)+\partial_{u_{2}} \psi(0)},
\end{aligned}
$$

whose inverse is $(5.4)$. Note that $\rho(\cdot)$ is a bijection from $(-1,1) \times(0, \infty)$ to the triangle $\mathbb{T}$ defined just after $(2.1)$.

Using (5.1) and (5.2) we see that the infinitesimal vector $(d x, d y, d z)$ (with $d z=$ $-\left(n_{1} d x+n_{2} d y\right) / n_{3}$ in view of (5.5) $)$ corresponds in the $P_{111}$ coordinates to

$$
\left(d u_{1}, d u_{2}\right)=\left(\frac{n_{3}+n_{2}}{n_{3}} d x+\frac{n_{2}}{n_{3}} d y, \frac{n_{1}}{n_{3}} d x+\frac{n_{2}+n_{3}}{n_{3}} d y\right)
$$

and in the $P_{110}$ coordinates to

$$
\left(d v_{1}, d v_{2}\right)=\left(d y-d x,-\frac{n_{1} d x+n_{2} d y}{n_{3}}\right) .
$$

Therefore, the ratio of area elements $d u / d v$ equals (using $n_{1}+n_{2}+n_{3}=1$ and $n_{1}=$ $\partial_{u_{1}} \psi, n_{2}=\partial_{u_{2}} \psi$ from $(5.5)$

$$
\frac{d u}{d v}=\frac{1}{n_{3}} \times \frac{n_{3}}{n_{1}+n_{2}}=\frac{1}{n_{1}+n_{2}}=\frac{1}{\partial_{u_{1}} \psi+\partial_{u_{2}} \psi}
$$

which is what we claim in $(5.3)$. 
5.1.1. Relation between $\sigma$ and $\hat{\sigma}$. We can now write

$$
\begin{aligned}
F(\psi) & =\int_{U} \sigma(\nabla \psi) d u=\int_{V} \sigma(\rho(\nabla \hat{\psi}(v))) \frac{d v}{\partial_{u_{1}} \psi(u(v))+\partial_{u_{2}} \psi(u(v))} \\
& =\int_{V} \sigma(\rho(\nabla \hat{\psi}(v)))\left(1+\frac{1}{2} \partial_{v_{2}} \hat{\psi}(v)\right) d v
\end{aligned}
$$

where in the last equality we used (5.4). This shows that the surface tension functional can also be expressed in the parametrization of the surface by $\hat{\psi}$ and that we only need to replace $\sigma(\nabla \psi)$ by $\hat{\sigma}(\nabla \hat{\psi})=\sigma(\rho(\nabla \hat{\psi}))\left(1+\frac{1}{2} \partial_{v_{2}} \hat{\psi}\right)$ as per (3.39).

5.1.2. Identity between (3.3) and (3.41). Here we verify that the equations (3.3) and (3.41) (together with 3.12$)$ ) for the hydrodynamic limit in the $\psi$ and $\hat{\psi}$ parametrization indeed describe the same interface evolution. Indeed, (3.3) and 3.41) can be rewritten as

$$
\partial_{t} \psi(u, t)=-\left.\mu(\nabla \psi(u, t)) \frac{\delta F}{\delta \psi(u)}\right|_{\psi(\cdot):=\psi(\cdot, t)}
$$

and

$$
\partial_{t} \hat{\psi}(v, t)=-\left.\hat{\mu}(\nabla \hat{\psi}(v, t)) \frac{\delta \hat{F}}{\delta \hat{\psi}(v)}\right|_{\hat{\psi}(\cdot):=\hat{\psi}(\cdot, t)}
$$

where $F(\psi)=\int_{U} \sigma(\nabla \psi) d u, \hat{F}(\hat{\psi})=\int_{V} \hat{\sigma}(\nabla \hat{\psi}) d v=F(\psi)$ and $\hat{\mu}$ is given in 3.47.

One verifies by direct inspection that

$$
\frac{\mu(\nabla \psi(u, t))}{\hat{\mu}(s(\nabla \psi(v(u), t)))}=\frac{\partial_{u_{1}} \psi(u, t)+\partial_{u_{2}} \psi(u, t)}{4},
$$

where $s(\rho)$ is the inverse of the function $\rho(s)=\left(\rho_{1}(s), \rho_{2}(s)\right)$ defined in 3.33) or 5.4). Next, we will prove that

$$
\frac{\partial_{t} \psi(u, t)}{\left.\partial_{t} \hat{\psi}(v, t)\right|_{v=v(u)}}=\frac{\partial_{u_{1}} \psi(u, t)+\partial_{u_{2}} \psi(u, t)}{2} .
$$

and that

$$
\frac{\delta F}{\delta \psi(u)}=\left.2 \frac{\delta \hat{F}}{\delta \hat{\psi}(v)}\right|_{v=v(u)} .
$$

Together with (5.11), these imply that (5.9) and (5.10) are indeed equivalent.

Let us verify (5.12). If $\psi(\cdot, t), \hat{\psi}(\cdot, t)$ are the height function and level set function of the same evolving surface $\Gamma_{t} \subset \mathbb{R}^{3}$, we want to find the relation between $\partial_{t} \psi(u, t)$ and $\partial_{t} \hat{\psi}(v, t)$. Let $p \in \Gamma_{t}$ and let the interface normal velocity $p$ be $V \times\left(n_{1}, n_{2}, n_{3}\right)$, with $\left(n_{1}, n_{2}, n_{3}\right)$ the normal vector normalized as $n_{1}+n_{2}+n_{3}=1$. Let the $\Pi_{111}$ and $\Pi_{110}$ projections of $p$ have coordinates $u_{0}$ and $v_{0}$ respectively. In analogy with (5.6), one has

$$
\begin{aligned}
& \psi\left(u_{0}+u, t+\epsilon\right)=-V^{\prime} \epsilon+n_{1} u_{1}+n_{2} u_{2}+O\left(\epsilon^{2}\right)+O\left(|u|^{2}\right)+C\left(u_{0}\right) \\
& \hat{\psi}\left(v_{0}+v, t+\epsilon\right)=\frac{-2 V^{\prime} \epsilon+v_{1}\left(n_{2}-n_{1}\right)+2 v_{2} n_{3}}{n_{1}+n_{2}}+O\left(\epsilon^{2}\right)+O\left(|v|^{2}\right)+C^{\prime}\left(v_{0}\right),
\end{aligned}
$$


with $V^{\prime}=\left(n_{1}^{2}+n_{2}^{2}+n_{3}^{2}\right) V$ and $C\left(u_{0}\right), C^{\prime}\left(v_{0}\right)$ independent of time and of $u, v$, whence

$$
\frac{\partial_{t} \psi\left(u_{0}, t\right)}{\partial_{t} \hat{\psi}\left(v_{0}, t\right)}=\frac{n_{1}+n_{2}}{2}=\frac{\partial_{u_{1}} \psi\left(u_{0}, t\right)+\partial_{u_{2}} \psi\left(u_{0}, t\right)}{2}
$$

and 5.12 follows.

It remains only to check (5.13). Under an infinitesimal variation $\psi \rightarrow \psi+\varepsilon \delta \psi$, the first variation of $F$ is

$$
\int_{U} \delta \psi(u) \frac{\delta F}{\delta \psi(u)} d u
$$

The same considerations (steps (5.14) and (5.15) that lead to (5.12) show that

$$
\delta \psi(u)=\delta \hat{\psi}(v(u)) \frac{\partial_{u_{1}} \psi(u)+\partial_{u_{2}} \psi(u)}{2} .
$$

Then, together with (5.3), the quantity in (5.16) can be rewritten as

$$
\left.\frac{1}{2} \int_{V} \frac{\delta F}{\delta \psi(u)}\right|_{u=u(v)} \delta \hat{\psi}(v) d v
$$

Since by construction $F(\psi)=\hat{F}(\hat{\psi})$, (5.17) implies (5.13).

5.2. Derivation of $(3.32)$. We will make an assumption of local equilibrium, namely, we assume that the law of $\eta\left(L^{2} t\right)$ in the neighborhood of any site $u_{L} \in U_{L}$, such that $\bar{u}=\lim _{L \rightarrow \infty} u_{L} / L$ is in the interior of $U$, approaches (when $L \rightarrow \infty$ ) the equilibrium measure $\pi_{\rho}$ with slope $\rho=\left(\partial_{u_{1}} \psi(u, t), \partial_{u_{2}} \psi(u, t)\right)=\rho(\nabla \hat{\psi}(v(u), t))$, where the function $\rho(\cdot)$ is defined in $(3.33)$.

Let us proceed to the derivation of $(3.32)$ under such assumption. Let $f: V \mapsto \mathbb{R}$ be a $C^{\infty}$ test function whose compact support is at positive distance from $\partial V$. We define for any configuration $\eta$

$$
I_{f}^{(L)}(\eta):=\frac{1}{\left|V_{L}\right|} \sum_{v \in V_{L}} f(v / L) \frac{\hat{h}_{\eta}(v)}{L},
$$

with $\left|V_{L}\right|=O\left(L^{2}\right)$ the cardinality of $V_{L}$. We will argue that

$$
\partial_{t} \mathbb{E}\left(I_{f}^{(L)}\left(\eta\left(L^{2} t\right)\right)\right) \stackrel{L \rightarrow \infty}{\rightarrow} \int_{V}\left[\partial_{v_{1}}^{2} f(v) \frac{\hat{\psi}(v, t)}{2}+\partial_{v_{2}} f(v)\left(1+\frac{\partial_{v_{2}} \hat{\psi}}{2}\right) \pi_{\rho(\nabla \hat{\psi}(v, t))}(A)\right] d v
$$

with

$$
A=A(\eta)=1_{X(0,0)} 1_{n\left(b^{(0,0)}\right) \neq 0} \frac{\left|n\left(b^{(0,0)}\right)\right|-1}{2}
$$

so that, at least in a weak sense, 3.32 must hold. Note also that in $(5.19)$ we are just looking at the average interface evolution. For an actual proof of the hydrodynamic limit, one should also prove that $I_{f}^{(L)}\left(\eta\left(L^{2} t\right)\right)-I_{f}^{(L)}(\eta(0))$ concentrates for $L \rightarrow \infty$ at the time-integral of the r.h.s. of (5.19).

The l.h.s. of $(5.19)$ is

$$
L^{2} \mathbb{E}\left(\left[\mathcal{L} I_{f}^{(L)}\right]\left(\eta\left(L^{2} t\right)\right)\right)
$$


with $\mathcal{L}$ the generator of the dynamics. We will work with Dynamics I (Definition 7 ) and leave to the reader to check that the same result is obtained with Dynamics II.

We need a couple of definitions. Given $v \in V_{L}$ and a configuration $\eta$, let

$$
r(\eta, v)=1_{\left(\Delta_{v_{1}} \hat{h}_{\eta}\right)(v) \neq 0}=\frac{1}{2}\left|\left(\Delta_{v_{1}} \hat{h}_{\eta}\right)(v)\right|,
$$

with $\Delta_{v_{1}}$ the discrete Laplacian in the direction $v_{1}:\left(\Delta_{v_{1}} \hat{h}_{\eta}\right)(v)=\hat{h}_{\eta}(v+(1,0))+\hat{h}_{\eta}(v-$ $(1,0))-2 \hat{h}_{\eta}(v)$, as well as

$$
\epsilon(\eta, v)=r(\eta, v) \operatorname{sign}\left[\left(\Delta_{v_{1}} \hat{h}_{\eta}\right)(v)\right]=\frac{1}{2}\left(\Delta_{v_{1}} \hat{h}_{\eta}\right)(v)
$$

and

$$
k(\eta, v)=r(\eta, v) \min \left\{n \geq 1: \hat{h}_{\eta}(v+\epsilon(\eta, v)(0, n)) \neq \hat{h}_{\eta}(v)\right\} .
$$

In words, $r\left(\eta,\left(v_{1}, v_{2}\right)\right)$ is the indicator function that the function $v_{1} \mapsto S^{\left(v_{2}\right)}\left(v_{1}\right)$ has a local extremum at $v_{1}, \epsilon(\eta, v)$ is -1 or +1 if the extremum is a maximum or minimum respectively and $k(\eta, v)$ is the number of minima or maxima that need to be flipped in order to reverse the minimum/maximum of $S^{\left(v_{2}\right)}(\cdot)$ into a maximum/minimum, see also Figure 8 .

Recall Definition 7 of the dynamics which, with the present notations, reads as follows: for any $v \in V_{L}$ such that $\epsilon(\eta, v) \neq 0$, with rate $1 /(2 k(\eta, v))$ all the heights $\hat{h}(v+$ $(0, n) \epsilon(\eta, v)), 0 \leq n<k(\eta, v)$ change by $+2 \epsilon(\eta, v)$. Therefore we have

$$
\begin{gathered}
L^{2}\left[\mathcal{L}^{I} I_{f}^{(L)}\right](\eta)=\frac{L}{\left|V_{L}\right|} \sum_{v \in V_{L}} r(\eta, v) \frac{2 \epsilon(\eta, v)}{2 k(\eta, v)} \sum_{n=0}^{k(\eta, v)-1} f\left(\frac{v_{1}}{L}, \frac{v_{2}+\epsilon(\eta, v) n}{L}\right) \\
=\frac{L}{\left|V_{L}\right|} \sum_{v \in V_{L}} \frac{1}{2}\left[\Delta_{v_{1}} \hat{h}_{\eta}\right](v) f(v / L) \\
+\frac{L}{\left|V_{L}\right|} \sum_{v \in V_{L}} r(\eta, v) \frac{\epsilon(\eta, v)}{k(\eta, v)} \sum_{n=0}^{k(\eta, v)-1}\left(f\left(\frac{v_{1}}{L}, \frac{v_{2}+\epsilon(\eta, v) n}{L}\right)-f\left(\frac{v}{L}\right)\right) .
\end{gathered}
$$

The sum in (5.25) can be rewritten, via a summation by parts and using $\Delta_{v_{1}} f(v / L)=$ $L^{-2} \partial_{v_{1}}^{2} f(v / L)+O\left(L^{-3}\right)$, as

$$
\frac{1}{2} \frac{1}{\left|V_{L}\right|} \sum_{v \in V_{L}} \frac{\hat{h}_{\eta}(v)}{L} \partial_{v_{1}}^{2} f(v / L)+O(1 / L)
$$

and the error term is uniform w.r.t. $\eta$. There are no boundary terms in the summation by parts because $f$ is compactly supported in the interior of $V$.

As for (5.26), let us Taylor expand

$$
f\left(\frac{v_{1}}{L}, \frac{v_{2}+\epsilon(\eta, v) n}{L}\right)-f\left(\frac{v}{L}\right)=\frac{\epsilon(\eta, v)}{L} n \partial_{v_{2}} f(v / L)+O\left(\left(\frac{k(\eta, v)}{L}\right)^{2}\right) .
$$


Altogether we get

$$
\begin{aligned}
\partial_{t} \mathbb{E}\left(I_{f}^{(L)}\left(\eta\left(L^{2} t\right)\right)\right) & =L^{2} \mathbb{E}\left[\mathcal{L}^{I} I_{f}\right]\left(\eta\left(L^{2} t\right)\right)=\frac{1}{2} \frac{1}{\left|V_{L}\right|} \sum_{v \in V_{L}} \frac{\mathbb{E} \hat{h}_{\eta\left(L^{2} t\right)}(v)}{L} \partial_{v_{1}}^{2} f(v / L) \\
& +\frac{1}{\left|V_{L}\right|} \sum_{v \in V_{L}} \partial_{v_{2}} f(v / L) \mathbb{E}\left[r\left(\eta\left(L^{2} t\right), v\right) \frac{k\left(\eta\left(L^{2} t\right), v\right)-1}{2}\right] \\
& +O\left(\frac{1}{L\left|V_{L}\right|} \sum_{v \in V_{L}} \mathbb{E} r\left(\eta\left(L^{2} t\right), v\right) k^{2}\left(\eta\left(L^{2} t\right), v\right)\right)+O(1 / L) .
\end{aligned}
$$

The first sum in the r.h.s. tends to

$$
\frac{1}{2} \int_{V} \hat{\psi}(v, t) \partial_{v_{1}}^{2} f(v) d v:
$$

just write the height $h_{\eta}(v)$ as the height at a point $v_{0}$ on the boundary of $V_{L}$ plus the sum of interface gradients from $v_{0}$ to $v$, then use the hypothesis of local equilibrium to replace the average interface gradients in terms of derivatives of $\hat{\psi}$.

As for the second term, we claim that under the assumption of local equilibrium we can replace

$$
\frac{1}{\left|V_{L}\right|} \sum_{v \in V_{L}} \partial_{v_{2}} f(v / L) \mathbb{E}\left[r\left(\eta\left(L^{2} t\right), v\right) \frac{k\left(\eta\left(L^{2} t\right), v\right)-1}{2}\right]
$$

with

$$
\int_{V} \partial_{v_{2}} f(v)\left(1+\frac{1}{2} \partial_{v_{2}} \hat{\psi}(v, t)\right) \pi_{\rho(\nabla \hat{\psi}(v, t))}\left[1_{X(0,0)} 1_{n\left(b^{(0,0)}\right) \neq 0} \frac{\left|n\left(b^{(0,0)}\right)\right|-1}{2}\right] d v .
$$

Let us see why. Let $v_{L} \in V_{L}$ be such that $v_{L} / L \rightarrow \bar{v}$ in the interior of $V$ and let $V_{\epsilon, L}$ be a ball of radius $\epsilon L$ around $v_{L}$. If $\Sigma_{\epsilon, L}$ is the portion of the interface $\Sigma$ whose $\Pi_{110}$ projection is $V_{\epsilon, L}$, let $U_{\epsilon, L}$ be its $\Pi_{111}$ projection. Then we have (with $\eta \equiv \eta\left(L^{2} t\right)$ )

$$
\begin{aligned}
& \frac{1}{\left|V_{\epsilon, L}\right|} \sum_{v \in V_{\epsilon, L}} r(\eta, v) \frac{k(\eta, v)-1}{2} \\
& \quad=\frac{\left|U_{\epsilon, L}\right|}{\left|V_{\epsilon, L}\right|} \times \frac{1}{\left|U_{\epsilon, L}\right|} \sum_{u \in U_{\epsilon, L}} 1_{X(u)} 1_{n\left(b^{(u)}\right) \neq\left(u_{1}+u_{2}\right) / 2} \frac{\left|n\left(b^{(u)}\right)-\left(u_{1}+u_{2}\right) / 2\right|-1}{2} .
\end{aligned}
$$

Indeed, the event $X(u) \cap\left\{n\left(b^{(u)}\right) \neq\left(u_{1}+u_{2}\right) / 2\right\}$ is the event that the edge $u, u+(1,1)$ is the common side of a lozenge of type 1 and one of type 2, see Definition 6 . In turn, this condition says that the point $u$ corresponds to one of the values of $v \in V_{L}$ with $r(\eta, v) \neq 0$, see Figure 8 .

Since $\left|U_{\epsilon, L}\right| /\left|V_{\epsilon, L}\right|$ equals the ratio between the total number of faces of $\Sigma_{\epsilon, L}$ and the number of its non-horizontal faces, by (5.3) and (5.4) this ratio converges to $[1+$ $\left.\frac{1}{2} \partial_{v_{2}} \hat{\psi}(\bar{v}, t)\right]$. As for the second term in (5.33), by the assumption of local equilibrium and the translation invariance of the Gibbs measures $\pi_{\rho}$, its average converges to the average in 5.32. 


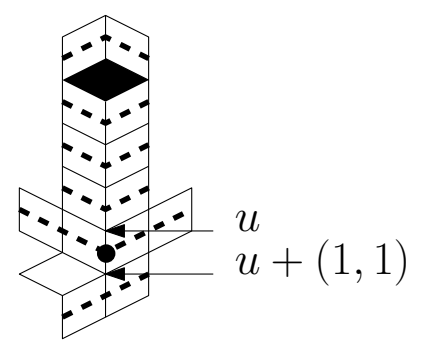

Figure 8 . The edge $u, u+(1,1)$ is common to a type- 1 and a type- 2 lozenge iff a level line $S^{\left(v_{2}\right)}$ (dashed) runs through the point $p$ (dot in the picture) at distance $1 / 2$ below $u$, and $S^{\left(v_{2}\right)}(\cdot)$ has a maximum/minimum at $v_{1}$ (a minimum in this example). Here, $\left(v_{1}, v_{2}\right)$ is the $\Pi_{110}$ projection of the point $p$. The distance $\left|n^{(u)}-\left(u_{1}+u_{2}\right) / 2\right|$ (=4 in the drawing) equals also $k\left(\eta,\left(v_{1}, v_{2}\right)\right)$. One sees that $k\left(\eta,\left(v_{1}, v_{2}\right)\right)$ is also the minimal value of $n \geq 1$ such that $\hat{h}\left(v_{1}, v_{2}+n\right) \neq \hat{h}\left(v_{1}, v_{2}\right)$, or the number of minima of the level set function that need to be flipped in order to flip the minimum at $v$.

Similarly to 5.32), one can replace

$$
\frac{1}{L\left|V_{L}\right|} \sum_{v \in V_{L}} \mathbb{E} r\left(\eta\left(L^{2} t\right), v\right) k^{2}\left(\eta\left(L^{2} t\right), v\right)
$$

with

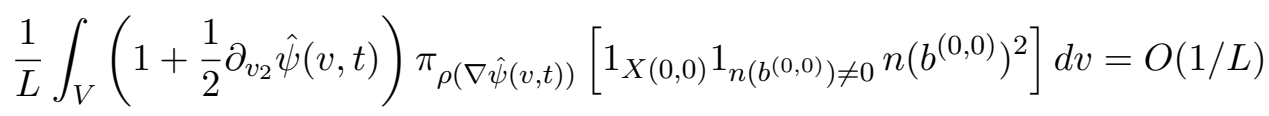

(the random variable $n\left(b^{(0,0)}\right)$ has moments of all orders under $\left.\pi_{\rho}\right)$. Altogether, (5.19) follows.

Remark 17. To justify that (5.34) is o(1) it would be clearly sufficient to prove that the second moment of $k(\eta, v)$ during the evolution remains $o(L)$. Under the equilibrium Gibbs measures $\pi_{\rho}$ the random variable $k(\eta, v)$ is known to have exponential moments of all orders, so the situation looks promising.

However, justifying the replacement of (5.31) with (5.32) looks definitely harder: following the $H^{-1}$ method of [6], it appears that one needs some form of uniform integrability bound on $k(\eta, v)$, uniformly in $L$. In the forthcoming work [12], in the case of periodic boundary conditions we manage to bypass this difficulty.

\section{Some Equilibrium computations}

6.1. Proof of Proposition 15. We begin with proving Eq. (3.34). From the last remark in Definition 6 we see that the event $X(0,0) \cap\left\{n\left(b^{(0,0)}\right) \neq 0\right\}$ is equivalently the event that the edge $e$ joining vertices $(0,0)$ and $(1,1)$ of $\mathcal{T}$ is the common side of two lozenges, one of type 1 and one of type 2. From (2.4) we see that (see Figure 4 for 
notations)

$$
\begin{aligned}
& \pi_{\rho}\left(X(0,0) \cap\left\{n\left(b^{(0,0)}\right) \neq 0\right\}\right) \\
& \quad=2 k_{1} k_{2}\left[K^{-1}\left(w_{1}, b_{1}\right) K^{-1}\left(w_{2}, b_{2}\right)-K^{-1}\left(w_{1}, b_{2}\right) K^{-1}\left(w_{2}, b_{1}\right)\right],
\end{aligned}
$$

where the factor 2 comes from the choice of whether the type- 1 lozenge is to the right or to the left of the edge $e$. From (2.6), we see that $k_{1} K^{-1}\left(w_{1}, b_{1}\right)=\rho_{1}, k_{2} K^{-1}\left(w_{2}, b_{2}\right)=$ $\rho_{2}, k_{3} K^{-1}\left(w_{1}, b_{2}\right)=\left(1-\rho_{1}-\rho_{2}\right)$. As a consequence,

$$
\begin{gathered}
\pi_{\rho}\left(X(0,0) \cap\left\{n\left(b^{(u)}\right) \neq 0\right\}\right)=2\left(\rho_{1} \rho_{2}+\left(1-\rho_{1}-\rho_{2}\right) \times \Xi\right) \\
\Xi=-\frac{k_{1} k_{2}}{k_{3}} \frac{1}{(2 \pi i)^{2}} \oint d z \oint d w \frac{1}{k_{3}+k_{1} z+k_{2} w} .
\end{gathered}
$$

Thanks to [4, Theorem 2.7] 7 , we know that $\Xi$ equals exactly $V(\rho)$.

Next we prove (3.35). For lightness of notation, we write $n, n^{+}, n^{-}, X,|I|$ instead of $n\left(b^{(0,0)}\right), n^{+}\left(b^{(0,0)}\right), n^{-}\left(b^{(0,0)}\right), X(0,0),\left|I\left(b^{(0,0)}\right)\right|$.

Remark 18. Since $\pi_{\rho}$ is translation-invariant and is locally the uniform measure on lozenge tilings, we have:

(U1) conditionally on the event $X$ and on the values of $n^{+}, n^{-}$, the random variable $n$ is uniformly distributed in $\left\{n^{-}, \ldots, n^{+}\right\}$.

(U2) conditionally on the event $X$ and on $n^{+-} n^{-}$, the random variable $n^{+}$is uniformly distributed on $\left\{0, \ldots, n^{+}-n^{-}\right\}$.

We want to compute

$$
\pi_{\rho}\left((|n|-1) 1_{X} 1_{n \neq 0}\right)=\pi_{\rho}\left(|n| 1_{X} 1_{n \neq 0}\right)-\pi_{\rho}\left(1_{X} 1_{n \neq 0}\right) .
$$

We have

$$
\pi_{\rho}\left(|n| 1_{X} 1_{n \neq 0}\right)=\pi_{\rho}\left(|n| 1_{X}\right)=\frac{1}{3} \pi_{\rho}\left(\frac{|I|^{2}-1}{|I|} 1_{X}\right) .
$$

Indeed, from (U1)-(U2) we see that, conditionally on $|I|:=n^{+}-n^{-}+1$, the two random variables $n^{+}$and $n^{+}-n$ are independent and uniformly distributed in $\{0, \ldots,|I|-1\}$. We have already mentioned in Section 4 that if $u_{1}, u_{2}$ are independent and uniform in $\{0, \ldots,|I|-1\}$ then

$$
E\left(\left|u_{1}-u_{2}\right|\right)=\frac{1}{3} \frac{|I|^{2}-1}{|I|} .
$$

Using (U1), the last expression in (6.4) is also

$$
\frac{1}{3} \pi_{\rho}\left((|I|+1) 1_{X} 1_{n \neq 0}\right)=2 V(\rho)
$$

where in the last step we used Theorem 11 .

On the other hand, $\pi_{\rho}\left(1_{X} 1_{n \neq 0}\right)$ was computed in (3.34). Altogether (6.3) equals

$$
2\left[-\rho_{1} \rho_{2}+\left(\rho_{1}+\rho_{2}\right) V(\rho)\right]
$$

and $(3.35)$ is proven.

\footnotetext{
${ }^{7}$ In the notations of [4, $k_{1}=b, k_{2}=c$ and $k_{1}=a$
} 
6.2. Proof of Theorem 11. Again, we write $n, n^{+}, n^{-}, X$ instead of $n\left(b^{(0,0)}\right), n^{+}\left(b^{(0,0)}\right)$, $n^{-}\left(b^{(0,0)}\right), X(0,0)$. Then, Eq. (3.11) together with definition 3.9$)$ gives

$$
\Delta:=\pi_{\rho}\left[\left(n^{+}+1\right) 1_{X} 1_{n<0}\right]=\frac{1}{\pi} \frac{\sin \left(\pi \rho_{1}\right) \sin \left(\pi \rho_{2}\right)}{\sin \left(\pi\left(1-\rho_{1}-\rho_{2}\right)\right)}
$$

and we need to prove that this equals

$$
\frac{1}{6} \pi_{\rho}\left[\left(n^{+}-n^{-}+2\right) 1_{X} 1_{n \neq 0}\right]
$$

which is twice the r.h.s. of (3.8).

The Gibbs measures $\pi_{\rho}$ are invariant by reflection through any vertex of $\mathcal{T}$ (see Remark 3.3 in [19]). As a consequence of this symmetry, conditionally on $X$, the random variables $n^{+} \geq 0$ and $-n^{-} \geq 0$ have the same law and

$$
\Delta=\pi_{\rho}\left[\left(1-n^{-}\right) 1_{X} 1_{n>0}\right] .
$$

We deduce from property (U1) above that

$$
2 \Delta=\pi_{\rho}\left[\left(1-n^{-}\right) \frac{n^{+}}{n^{+}-n^{-}+1}+\left(1+n^{+}\right) \frac{\left|n^{-}\right|}{n^{+}-n^{-}+1} ; X\right] .
$$

In fact, by first conditioning on the event $X$ and then on the value of $n^{+}, n^{-}$and using that $n$ is conditionally uniform on $\left\{n^{-}, \ldots, n^{+}\right\}$one has (with $\pi_{\rho}^{X}$ the measure $\pi_{\rho}$ conditioned to the event $X$ )

$$
\begin{aligned}
& \pi_{\rho}\left[\left(n^{+}+1\right) 1_{X} 1_{n<0}\right]=\pi_{\rho}^{X}\left[\left(n^{+}+1\right) 1_{n<0}\right] \pi_{\rho}(X) \\
& \begin{aligned}
=\pi_{\rho}^{X}\left[\left(n^{+}+1\right) \pi_{\rho}^{X}\left(1_{n<0} \mid n^{+}, n^{-}\right)\right] \pi_{\rho}(X)= & \pi_{\rho}^{X}\left[\left(n^{+}+1\right) \frac{\left|n^{-}\right|}{n^{+}-n^{-}+1}\right] \pi_{\rho}(X) \\
& =\pi_{\rho}\left[\left(n^{+}+1\right) \frac{\left|n^{-}\right|}{n^{+}-n^{-}+1}\right],
\end{aligned}
\end{aligned}
$$

and similarly for $\pi_{\rho}\left[\left(1-n^{-}\right) 1_{X} 1_{n>0}\right]$. Using property (U2) (together with $E U=$ $k / 2, E U^{2}=(1 / 6) k(1+2 k)$ for a uniform random variable $U$ on $\left.\{0, \ldots, k\}\right)$ we have

$$
\pi_{\rho}\left[n^{+} n^{-} \mid X, n^{+}-n^{-}\right]=-\frac{1}{6}\left(n^{+}-n^{-}\right)^{2}+\frac{1}{6}\left(n^{+}-n^{-}\right) .
$$

As a consequence,

$$
\Delta=\frac{1}{6} \pi_{\rho}\left[\frac{\left(n^{+}-n^{-}\right)^{2}+2\left(n^{+}-n^{-}\right)}{n^{+}-n^{-}+1} ; X\right]=\frac{1}{6} \pi_{\rho}\left[\left(n^{+}-n^{-}+2\right) \frac{n^{+}-n^{-}}{n^{+}-n^{-}+1} ; X\right] .
$$

On the other hand, from (U2), this equals 6.8).

\section{ACKNOWLEDGMENTS}

We would like to thank the anonymous referee for a careful reading. We are grateful to Pietro Caputo and Fabio Martinelli for innumerable enlightening discussions. F. T. was partially funded by Marie Curie IEF Action DMCP "Dimers, Markov chains and Critical Phenomena", grant agreement n. 621894 and by the CNRS PICS grant 
Interfaces aléatoires discrètes et dynamiques de Glauber. B. Laslier was supported by the Engineering and Physical Sciences Research Council under grant EP/103372X/1.

\section{REFERENCES}

[1] Y. Akutsu, N. Akutsu, T. Yamamoto, Universal jump of Gaussian curvature at the facet edge of a crystal, Phys. Rev. Lett. 61 (1988), 424-427.

[2] P. Caputo, F. Martinelli, F. Simenhaus, F. L. Toninelli, "Zero" temperature stochastic 3D Ising model and dimer covering fluctuations: a first step towards interface mean curvature motion, Comm. Pure Appl. Math. 64 (2011), 778-831.

[3] P. Caputo, F. Martinelli, F. L. Toninelli, Mixing times of monotone surfaces and SOS interfaces: a mean curvature approach, Comm. Math. Phys. 311 (2012), 157-189

[4] S. Chhita, P. L. Ferrari, A combinatorial identity for the speed of growth in an anisotropic KPZ model, arXiv:1508.01665 to appear on Ann. Inst. Henri Poincaré D.

[5] H. Cohn, R. Kenyon, J. Propp, A variational principle for domino tilings, J. Amer. Math. Soc. 14 (2001), 297-346

[6] T. Funaki, H. Spohn, Motion by Mean Curvature from the Ginzburg-Landau $\nabla \phi$ Interface Model, Comm. Math. Phys. 85 (1997), 136

[7] T. Funaki, Stochastic interface models. Lectures on probability theory and statistics, 103-274, Lecture Notes in Math., 1869, Springer, Berlin, 2005.

[8] R. Kenyon, Lectures on dimers, Statistical mechanics, 191-230, IAS/Park City Math. Ser., 16 Amer. Math. Soc., Providence, RI, 2009

[9] R. Kenyon, A. Okounkov, S. Sheffield, Dimers and amoebae, Ann. Math. 163 (2006), 1019-1056.

[10] C. Kipnis, C. Landim, Scaling Limits of Interacting Particle Systems, Springer, 1999.

[11] B. Laslier, F. L. Toninelli, Lozenge tilings, Glauber dynamics and macroscopic shape, Comm. Math. Phys. 338 (2015), 1287-1326.

[12] B. Laslier, F. L. Toninelli, Lozenge tiling dynamics and convergence to the hydrodynamic equation, work in progress.

[13] G. M. Lieberman, Second order parabolic differential equations, World Scientific, 1996

[14] M. Luby, D. Randall, A. Sinclair, Markov Chain Algorithms for Planar Lattice Structures, SIAM J. Comput. 31 (2001), 167-192.

[15] T. Nishikawa, Hydrodynamic limit for the Ginzburg-Landau $\nabla \phi$ interface model with boundary conditions, Comm. Math. Phys. 127 (2003), 205-227.

[16] H. Spohn, Interface motion in models with stochastic dynamics, J. Stat. Phys. 71 (1993), 1081-1132.

[17] M. H. Protter, H. F. Weinberger, Maximum principles in differential equations, Springer, New York, 1999.

[18] H. Spohn, Large Scale Dynamics of Interacting Particles, Springer, 1991.

[19] F. L. Toninelli, A (2+1)-dimensional growth process with explicit stationary measure, to appear on Ann. Probab., arXiv:1503.05339

[20] D. B. Wilson, Mixing times of lozenge tiling and card shuffling Markov chains, Ann. Appl. Probab. 14 (2004), 274-325.

Statslab, Centre for Mathematical Sciences, Wilberforce Road, Cambridge CB3 0WA - UK, and, LPMA - Univ. Paris Diderot, Btiment Sophie Germain, avenue de France, 75013

PARIS, France, E-MAIL: LASLIER@MATh.univ-PARIS-DIDERot.Fr

Univ Lyon 1, Université Claude Bernard Lyon 1, CNRS UMR 5208, Institut Camille Jor-

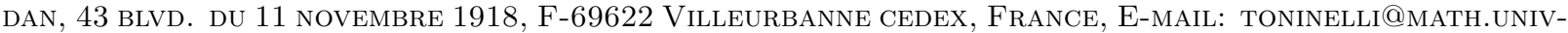
LYON1.FR 